\title{
THE LINDELÖF PRINCIPLE AND ANGULAR DERIVATIVES IN CONVEX DOMAINS OF FINITE TYPE
}

\author{
MARCO ABATE and ROBERTO TAURASO
}

(Received 26 February 2001; revised 5 September 2001)

\author{
Communicated by P. C. Fenton
}

\begin{abstract}
We describe a generalization of the classical Julia-Wolff-Carathéodory theorem to a large class of bounded convex domains of finite type, including convex circular domains and convex domains with real analytic boundary. The main tools used in the proofs are several explicit estimates on the boundary behaviour of Kobayashi distance and metric, and a new Lindelöf principle.
\end{abstract}

2000 Mathematics subject classification: primary 32A40; secondary $32 \mathrm{H} 40$.

\section{Introduction}

A classical result in the theory of one complex variable, due to Fatou [12], says that a bounded holomorphic function defined on the unit disk $\Delta$ in the complex plane admits non-tangential limit at a.e. point $\sigma \in \partial \Delta$. Clearly, this theorem leaves open the question of what happens at a specific point $\sigma_{0} \in \partial \Delta$. Of course, to get a sensible statement one needs to make some assumptions on the function $f$. In 1920, Julia ([18]) identified the right hypotheses, showing how to get the existence of the non-tangential limit at a given boundary point using Schwarz's lemma. But the real breakthrough is due to Wolff ([28]) in 1926 and Carathéodory ([8]) in 1929, who proved, under Julia's hypotheses, that the derivative too admits non-tangential limit at the specified boundary point. Their results are collected in the following statement, known as the Julia-Wolff-Carathéodory theorem.

THEOREM 0.1 (Julia-Wolff-Carathéodory). Let $f \in \operatorname{Hol}(\Delta, \Delta)$ and $\sigma_{0} \in \partial \Delta$ be such that

$$
\liminf _{\zeta \rightarrow \sigma_{0}} \frac{1-|f(\zeta)|}{1-|\zeta|}=\alpha<+\infty .
$$

(C) 2002 Australian Mathematical Society $1446-7887 / 2000 \$ A 2.00+0.00$ 
Then

(i) $f$ has non-tangential limit $\tau_{0} \in \partial \Delta$ at $\sigma_{0}$;

(ii) $f^{\prime}$ has non-tangential limit $\alpha \tau_{0} \bar{\sigma}_{0}$ at $\sigma_{0}$.

See, for example, [3] for proofs and applications. It should be remarked that the lim inf in (0.1) is always strictly positive.

The extension of this theorem to bounded holomorphic functions of several variables is clearly a natural problem. This has been done in several cases: the unit ball $B^{n}$ of $\mathbb{C}^{n}$ (Hervé [15] and Rudin [26]); strongly convex domains ([4]); strongly pseudoconvex domains ([5]); and polydisks (Jafari [16] and [6]). The aim of this paper is to describe a generalization of this theorem (and of a related result, the Lindelöf principle; see below) to a large class of bounded convex domains of finite type, including the convex circular domains and the convex domains with real analytic boundary. The spirit of the approach is the same as in [4] and [6] (see [7] for a more informal description of the background ideas and of the involved techniques); but the details of the proofs are different. In particular, the version of the Lindelöf principle we discuss here is both valid in more general domains and requires weaker hypotheses than the one proved, for instance, in [4].

The main idea behind these generalizations is that the boundary behaviour of holomorphic functions defined on (or with values in) a bounded domain must be controlled by the boundary behaviour of the intrinsic Kobayashi distance and metric of the domain. Therefore to compare our results with the classical ones we must translate the hypotheses of Theorem 0.1 in terms involving the Kobayashi metric and distance.

We begin with Theorem 0.1 . In the disk, $1-|\zeta|$ measures the euclidean distance of $\zeta$ from the boundary $\partial D$. Now, if $D \subset \subset \mathbb{C}^{n}$ is a bounded strongly pseudoconvex or convex domain, it is known that the Kobayashi distance $k_{D}\left(z_{0}, z\right)$ from a given point $z_{0} \in D$ goes to infinity exactly as $-(1 / 2) \log \delta(z)$ as $z$ tends to $\partial D$, where $\delta(z)$ is the euclidean distance of $z$ from the boundary $\partial D$. Then a sensible translation of $(0.1)$ for a bounded holomorphic function $f: D \rightarrow \Delta$ and a point $x \in \partial D$ is

$$
\liminf _{z \rightarrow x}\left[k_{D}\left(z_{0}, z\right)-k_{\Delta}(0, f(z))\right]<+\infty .
$$

Indeed, this is exactly what is needed to generalize Julia's part of the Julia-Wolff-Carathéodory theorem.

THEOREM 0.2 ([4]). Let $D \subset \subset \mathbb{C}^{n}$ be complete hyperbolic. Take $f \in \operatorname{Hol}(D, \Delta)$ and $x \in \partial D$ such that

$$
\liminf _{z \rightarrow x}\left[k_{D}\left(z_{0}, z\right)-k_{\Delta}(0, f(z))\right]<+\infty .
$$

Then $f$ has $K$-limit $\tau \in \partial \Delta$ at $x$. 
It is worth pointing out that similar statements hold for holomorphic maps $f: D_{1} \rightarrow$ $D_{2}$ under suitable hypotheses on $D_{2}$ (for example, if $D_{2}$ is bounded strongly pseudoconvex). The proof is just an application of the contracting property of the Kobayashi distance.

The $K$-limit appearing in the statement of Theorem 0.2 is a several variable generalization (one of many) of the notion of non-tangential limit. One way of defining the non-tangential limit in the unit disk is using Stolz regions: a Stolz region $K_{\Delta}(\sigma, M) \subset \Delta$ of vertex $\sigma \in \partial \Delta$ and amplitude $M>1$ is the egg-shaped region given by

$$
K_{\Delta}(\sigma, M)=\left\{\zeta \in \Delta \mid \frac{|\sigma-\zeta|}{1-|\zeta|}<M\right\}
$$

Then $f: \Delta \rightarrow \mathbb{C}$ has non-tangential limit $L \in \mathbb{C}$ at $\sigma$ if and only if $f(\zeta) \rightarrow L$ as $\zeta \rightarrow \sigma$ inside any Stolz region of vertex $\sigma$. Now, the work by Korányi and Stein on Fatou's theorem in strongly pseudoconvex domains has revealed that the right generalization of Stolz regions in several variable is not a cone-shaped region, but a region which is non-tangential only in the direction orthogonal to the boundary, and instead at least parabolically tangent to the boundary along the complex-tangential directions.

In [4] we discovered a way to define, using just the Kobayashi distance, an approach region for domains in several complex variables which is comparable to KorányiStein's: the $K$-region of vertex $x \in \partial D$, amplitude $M>1$ and pole $z_{0} \in D$ defined by setting

$$
K_{z_{0}}(x, M)=\left\{z \in D \mid \limsup _{w \rightarrow x}\left[k_{D}(z, w)-k_{D}\left(z_{0}, w\right)\right]+k_{D}\left(z_{0}, z\right)<\log M\right\} ;
$$

notice that changing the pole amounts to a shifting of the amplitudes, and so it is not relevant. The $K$-regions coincide with the Stolz regions in the unit disk (and with Korányi-Stein regions in the unit ball $B^{n}$ ), and are well-suited to interact with conditions like (0.2). We shall then say that a function $f: D \rightarrow \mathbb{C}$ admits $K$ limit $L \in \mathbb{C}$ at $x \in \partial D$ if $f(z) \rightarrow L$ as $z \rightarrow x$ inside any $K$-region of vertex $x$.

Unfortunately, as Rudin already remarked, in the unit ball the $K$-limit is a generalization of the non-tangential limit not suitable for extensions of the Wolff-Carathéodory part of Theorem 0.1 . The correct version is a somewhat weaker (and more technical) notion we call restricted $K$-limit, whose origin lies in the classical Lindelöf principle. This says that, given a point $\sigma \in \partial \Delta$, a bounded (or just bounded in Stolz regions with vertex at $x$ ) holomorphic function $f: \Delta \rightarrow \mathbb{C}$ admits non-tangential limit $L \in \mathbb{C}$ at $\sigma$ if and only if it admits limit $L$ when restricted to a single non-tangential curve $\gamma:[0,1) \rightarrow \Delta$ such that $\gamma(t) \rightarrow \sigma$ as $t \rightarrow 1^{-}$. 
Črka has been the first one to generalize the Lindelöf principle to bounded holomorphic functions of several complex variables, remarking that the correct statement involved, instead of the non-tangential limit, the existence of the limit along all curves in a suitable class including all non-tangential ones. In the case of strongly pseudoconvex domains, it amounted to curves that were non-tangential in the direction orthogonal to the boundary, and asymptotically less than parabolically tangent to the boundary along the complex-tangential directions; so the existence of the (same) limit along all these curves is slightly weaker than the existence of the $K$-limit, but stronger than the existence of the non-tangential limit.

In [4], inspired by [10], we described a general procedure to get new Lindelöf principles; unfortunately, it works only for bounded holomorphic functions, whereas the functions appearing in generalizations of the Wolff-Carathéodory part of Theorem 0.1 are in general only bounded in $K$-regions, with the bound depending on the amplitude of the region.

One then needs a Lindelöf principle for $K$-bounded (that is, bounded in $K$-regions) functions. Possibly the main new result of this paper is exactly such a Lindelöf principle, holding in any bounded convex domain of finite type under weaker hypotheses on $f$.

THEOREM 0.3. Let $D \subset \mathbb{C}^{n}$ be a bounded convex domain, and fix $z_{0} \in D$. Let $x \in \partial D$ such that $\partial D$ is smooth near $x$, and assume that the line type of $x$ is finite. Let $f \in \operatorname{Hol}(D, \mathbb{C})$ be $T$-bounded at $x \in \partial D$. If $f\left(\gamma^{o}(t)\right) \rightarrow L$ as $t \rightarrow 1^{-}$for a special restricted $x$-curve $\gamma^{o}$, then $f$ has restricted $K$-limit $L$ at $x$.

Here $T$-bounded means bounded in $T$-regions, which are approach regions smaller than $K$-regions but defined only in convex domains; an $x$-curve is a curve in $D$ ending at $x \in \partial D$; a restricted curve is, roughly speaking, a curve whose orthogonal projection into the complex line orthogonal to $\partial D$ in $x$ approaches $x$ non-tangentially (this is not the actual definition, but it has the correct flavour without relying on too many details); a special curve is a curve such that its Kobayashi distance from its projection tends to zero; and having restricted $K$-limit $L$ means having limit $L$ restricted to any special restricted curve (see Section 3 for the exact definitions).

We are finally able to state our generalization of Theorem 0.1 (ii), saying that the derivatives of a bounded holomorphic function satisfying (0.2) admits restricted $K$-limit, even with weights:

THEOREM 0.4. Let $D \subset \subset \mathbb{C}^{n}$ be a 'good' convex domain of finite type, and take $x \in \partial D$. Let $f \in \operatorname{Hol}(D, \Delta)$ be such that $(0.2)$ holds at $x$. For $v \in \mathbb{C}^{n}, v \neq O$, let $0<s \leq 1$ be such that $\delta(z)^{s} \kappa_{D}(z ; v)$ is T-bounded at $x$, where $\delta(z)$ is the euclidean distance from the boundary, and $\kappa_{D}$ is the Kobayashi metric of $D$. Then the function

$$
\delta(z)^{s-1} \partial f(z) / \partial v
$$


is $T$-bounded at $x$, and

(i) if $v$ is transversal to $T_{x}^{\mathrm{C}}(\partial D)$ then $s=1$, and $(0.4)$ has non-zero restricted $K$-limit at $x$;

(ii) if $v$ is complex-tangential to $\partial D$ at $x$ then $s<1$ and $(0.4)$ has restricted $K$-limit zero at $x$.

Thus the behaviour of the Kobayashi metric at the boundary controls the boundary behaviour of the derivatives of $f$, as anticipated at the beginning of this introduction. One final remark: by a 'good' convex domain of finite type here we mean a domain satisfying a couple of technical hypotheses needed in the proof (see the remarks at the end of Section 2 and Section 4 and the statement of Theorem 4.2 for the exact assumptions needed) but possibly not needed for the validity of the theorem. Anyway, we know that strongly convex domains, weakly convex domains with real analytic boundary and convex circular domains of finite type are 'good' in this sense, and thus Theorem 0.4 can be applied to a much larger class of domains than the corresponding results in [4].

This paper is organized as follows. Section 1 collects several estimates on the boundary behaviour of the Kobayashi distance and metric in convex domains of finite type, whose proof depends on McNeal's work [22,23]. Section 2 is devoted to the study of complex geodesics in convex domains of finite type, a technical tool we shall heavily need in the rest of the paper. Section 3 contains our new Lindelöf principle, and Section 4 the proof of Theorem 0.4 .

\section{Estimates}

Let $D=\left\{z \in \mathbb{C}^{n} \mid r(z)<0\right\} \subset \subset \mathbb{C}^{n}$ be a bounded domain, and $x \in \partial D$. If the boundary of $D$ is smooth nearby $x$, we say that $x$ is a point of finite line type if

$$
\sup \left\{v(r \circ l) \mid l: \mathbb{C} \rightarrow \mathbb{C}^{n} \text { is linear and } l(0)=x\right\}=L<+\infty \text {, }
$$

where $v(r \circ l)$ denote the order of vanishing of $r \circ l$ in $x$. The number $L$ is the maximum order of contact of $\partial D$ with complex lines in $x$; in particular, $L \geq 2$ (it suffices to consider a line tangent to $\partial D$ ). Furthermore, if $D$ is convex nearby $x$ then McNeal proved that $x$ is of finite line type if and only if it is of finite type in the sense of D'Angelo. For this reason we shall say that a bounded convex domain $D$ is simply of finite type $L<+\infty$ if the line type of all $x \in \partial D$ is bounded by $L$ (and the bound is achieved at some point). In particular, $D$ is strongly convex if and only if it is of finite type 2 .

In this section we collect several estimates on the boundary behaviour of the Kobayashi metric and distance on a convex domain of finite type we shall need in the 
sequel. We shall denote by $k_{D}(z, w)$ the Kobayashi distance of $z$ and $w$ in the domain $D$, and by $\kappa_{D}(z ; v)$ the Kobayashi length in $D$ of the tangent vector $v$ at the point $z$. We shall also consistently use the following notation: if $f$ and $g$ are functions, we shall write $f \lesssim g$ to mean that there is a constant $C$, sometimes universal sometimes depending on specified parameters but always independent of $f$ and $g$, such that $f \leq C g$. If $f \lesssim g$ and $g \lesssim f$, we shall write $f \approx g$.

For $q \in D$ and $v \in \mathbb{C}^{*}$, we denote by $\delta(q)=d(q, \partial D)$ the euclidean distance from $q$ to the boundary of $D$, and by $\delta(q ; v)$ the euclidean distance from $q$ to the boundary of the intersection of $D$ with the complex line through $q$ parallel to $v$. We shall also denote by $B\left(x_{0}, \varepsilon\right)$ the euclidean ball of center $x_{0}$ and radius $\varepsilon$.

We begin recalling two estimates which holds for any $C^{2}$ domain:

LEMMA 1.1. Let $D \subset \subset \mathbb{C}^{n}$ be a $C^{2}$ bounded domain.

(i) Given $z_{0} \in D$, there is a constant $c_{1}$ such that for all $z \in D$

$$
k_{D}\left(z_{0}, z\right) \leq c_{1}-\frac{1}{2} \log \delta(z) .
$$

(ii) Given $x_{0} \in \partial D$, there exist $\epsilon$ and $c>0$ such that for all $z_{1}, z_{2} \in D \cap B\left(x_{0}, \varepsilon\right)$ we have

$$
k_{D}\left(z_{1}, z_{2}\right) \leq \frac{1}{2} \sum_{j=1}^{2} \log \left(1+\frac{\left\|z_{1}-z_{2}\right\|}{\delta\left(z_{j}\right)}\right)+c .
$$

ProOF. (i) See [3, Theorem 2.3.51] or [1].

(ii) See [3, Theorem 2.3.56] or [13].

For the next estimate we need a new notation. Let $D \subset \subset \mathbb{C}^{n}$ be a bounded $C^{2}$ domain; in particular, there is $\varepsilon>0$ such that $\partial D$ admits a tubular neighbourhood $U$ of radius $\varepsilon$. For any $z \in U$, there is a closest $x=x(z) \in \partial D$; we then extend the exterior normal unit vector field $\mathbf{n}$ from $\partial D$ to $U$ by setting $\mathbf{n}_{z}=\mathbf{n}_{x(z)}$. If $z \in D \cap U$ and $v \in \mathbb{C}^{n}=T_{z} D$, we shall write $v=v_{N}+v_{T}$, where $v_{N}=\left(v, \mathbf{n}_{z}\right) \mathbf{n}_{z}$ is the normal component of $v$ at $z$ and $v_{\tau}=v-v_{N}$ is the tangential component of $v$ at $z$ (and $(\cdot, \cdot)$ denotes the canonical hermitian product of $\mathbb{C}^{n}$ ).

LEMMA 1.2. Let $D \subset \subset \mathbb{C}^{n}$ be a $C^{2}$ bounded domain. Then there are $\varepsilon>0$ and $c>0$ such that if $z \in D$ is such that $\delta(z)<\varepsilon$ and $v \in \mathbb{C}^{n}$ then

$$
\kappa_{D}(z ; v) \leq \frac{\left\|v_{N}\right\|}{\delta(z)}+c \frac{\left\|v_{T}\right\|}{\delta(z)^{1 / 2}} .
$$

PROOF. Let $\varepsilon>0$ be such that $\partial D$ admits a tubular neighbourhood of radius $2 \varepsilon$. In particular, for every $z \in D$ such that $\delta(z)<\varepsilon$ there is $z_{0} \in D$ such that $\delta\left(z_{0}\right)=\varepsilon$, 
$x\left(z_{0}\right)=x(z), \delta(z)=\varepsilon-\left\|z-z_{0}\right\|$ and the euclidean ball $B$ of center $z_{0}$ and radius $\varepsilon$ is contained in $D$ and tangent to $\partial D$ in $x\left(z_{0}\right)$. The decreasing property of the Kobayashi metric implies $\kappa_{D}(z ; v) \leq \kappa_{B}(z ; v)$; therefore it suffices to estimate the latter.

Now, it is easy to see that

therefore

$$
\kappa_{B}(z ; v)^{2}=\frac{\varepsilon^{2}}{\left(\varepsilon+\left\|z-z_{0}\right\|\right)^{2}} \frac{\left\|v_{N}\right\|^{2}}{\delta(z)^{2}}+\frac{\left\|v_{T}\right\|^{2}}{\delta(z)\left(\varepsilon+\left\|z-z_{0}\right\|\right)} ;
$$

$$
\kappa_{B}(z ; v)^{2} \leq \frac{\left\|v_{N}\right\|^{2}}{\delta(z)^{2}}+\frac{\left\|v_{T}\right\|^{2}}{\varepsilon \delta(z)},
$$

and we are done.

For the next couple of results we need the convexity of $D$ :

LEMMA 1.3. Let $D \subset \subset \mathbb{C}^{n}$ be a $C^{2}$ bounded convex domain. Then:

(i) Given $z_{0} \in D$, there is a constant $c_{2} \in \mathbb{R}$ such that for all $z \in D$

$$
c_{2}-\frac{1}{2} \log \delta(z) \leq k_{D}\left(z_{0}, z\right) .
$$

(ii) There is $\varepsilon>0$ such that if $z \in D$ is such that $\delta(z)<\varepsilon$ and $v \in \mathbb{C}^{n}$ then

$$
\frac{\left\|v_{N}\right\|}{\delta(z)} \lesssim \kappa_{D}(z ; v)
$$

where the constant depends only on $D$.

Proof. (i) The estimate (1.4) is known for strongly pseudoconvex domain (see [3, Theorem 2.3.52], [1, 24,27]); but we now show that it holds for weakly convex domains too. The real tangent plane to $\partial D$ at $x$ is given by $\operatorname{Re} \chi_{x}(z)=0$, where

$$
\chi_{x}(z)=\sum_{h=1}^{n} \frac{\partial r}{\partial z^{h}}(x)\left(x^{h}-z^{h}\right)
$$

and $r$ is a $C^{2}$ defining function for $D$.

Since $\partial D$ is compact and $C^{2}$, there is a constant $M$ depending only on $D$ such that $\left|\chi_{x}(z)\right| \leq M\|x-z\|$. If $H=\{\operatorname{Re} \zeta>0\} \subset \mathbb{C}$ is the right half-plane, by convexity we have $\chi_{x}(D) \subset H$ for any $x \in \partial D$. Choosing $x=x(z)$, the explicit expression of $k_{H}$ yields a $c_{2}^{\prime}>0$ depending only on $z_{0}$ and $D$ such that

$$
\begin{aligned}
k_{D}\left(z_{0}, z\right) & \geq k_{H}\left(\chi_{x(z)}\left(z_{0}\right), \chi_{x(z)}(z)\right) \geq c_{2}^{\prime}-\frac{1}{2} \log \operatorname{Re} \chi_{x(z)}(z) \\
& \geq c_{2}^{\prime}-\frac{1}{2} \log \left|\chi_{x(z)}(z)\right| \geq c_{2}-\frac{1}{2} \log \delta(z),
\end{aligned}
$$


where $c_{2}=c_{2}^{\prime}-\frac{1}{2} \log M$, because $\|x(z)-z\|=\delta(z)$ by construction.

(ii) As usual, let $\varepsilon>0$ be such that there is a tubular neighbourhood $U$ of $\partial D$ of radius $\varepsilon$. So take $z \in U \cap D$, and choose again $x=x(z) \in \partial D$. Then

$$
\kappa_{D}(z ; v) \geq \kappa_{H}\left(\chi_{x}(z) ; d \chi_{x}(v)\right)=\frac{\left|d \chi_{x}(v)\right|}{2 \operatorname{Re} \chi_{x}(z)} \geq \frac{\left|d \chi_{x}(v)\right|}{2 M \delta(z)} .
$$

On the other hand, the complex gradient of $r$ at $x \in \partial D$ is a non-vanishing real multiple of the conjugate of the exterior unit normal of $\partial D$ at $x$; therefore there is $M_{1}>0$ depending only on $D$ such that $\left(1 / M_{1}\right)\left|\left(v, \mathbf{n}_{x}\right)\right| \leq\left|d \chi_{x}(v)\right| \leq M_{1}\left|\left(v, \mathbf{n}_{x}\right)\right|$, and we are done, because $\mathbf{n}_{x}=\mathbf{n}_{z}$ by construction.

The final bunch of estimates depends on the finite type condition. To state them, we recall the following basic result by $\mathrm{McNeal}$.

THEOREM $1.4([22,23])$. Let $D=\left\{z \in \mathbb{C}^{n} \mid r(z)<0\right\}$ be a bounded domain, and let $x \in \partial D$. Assume that there is a neighbourhood $U$ of $x$ in $\mathbb{C}^{n}$ such that $D \cap U$ is convex and smooth near $x$; assume moreover that the line type of $x$ is $L<+\infty$. Then, after possibly shrinking $U$, for every $q \in D \cap U$ there exist an affine isometric change of coordinates, positive numbers $\tau_{1}=\delta(q) \leq \tau_{2} \leq \cdots \leq \tau_{n}$ and points $p_{1}, p_{2}, \ldots, p_{n} \in \partial D$ such that the new system of coordinates $\left(z^{1}, z^{2}, \ldots, z^{n}\right)$ is centered in $q$ and we have:

$$
\begin{aligned}
\partial r\left(p_{j}\right) / \partial z^{i}=0 ; & \text { for } i>j, \\
\delta(q) / \tau_{i} \lesssim\left|\partial r\left(p_{i}\right) / \partial z^{i}\right| \lesssim \delta(q) / \tau_{i} ; & \text { for } 1 \leq i \leq n, \\
\left|\partial r\left(p_{j}\right) / \partial z^{i}\right| \lesssim \delta(q) / \tau_{i} ; & \text { for } i<j, \\
\delta(q)^{1 / 2} \lesssim \tau_{j} \lesssim \delta(q)^{1 / L}, & \text { for } j=2, \ldots, n,
\end{aligned}
$$

where the constants are independent of $q$.

The construction of McNeal coordinates is as follows. Let $\tau_{1}$ be the distance from $q$ to $\partial D$, and $p_{1} \in \partial D$ a point where the distance is realized. Let $z^{1}$ be the affine isometric parametrization of the complex line from $q$ to $p_{1}$ with $z^{1}(0)=q$ and $p_{1}$ lying on the positive $\operatorname{Re} z^{1}$ axis. Now let $\tau_{2}$ be the distance from $q$ to the intersection of $\partial D$ with the (complex) orthogonal complement (through $q$ ) of the span of the coordinate $z_{1}$; we then choose $p_{2} \in \partial D$ to be a point where this distance is achieved, and as $z^{2}$ the affine isometric parametrization of the complex line from $q$ to $p_{2}$ with $z^{2}(q)=0$ and $p_{2}$ lying on the positive $\operatorname{Re} z^{2}$ axis. Continuing this process we get the $n$ coordinate functions satisfying the assertions of Theorem 1.4.

Using McNeal coordinates we can give a sort of local model for the boundary of a convex domain of finite type. 
LEMMA 1.5. Let $D \subset \mathbb{C}^{n}$ be a bounded convex domain. Let $x \in \partial D$ such that $\partial D$ is smooth near $x$, and assume that the type of $x$ is finite. Then there is a neighbourhood $U$ of $x$ such that for every $q \in D \cap U$ the domain

$$
A_{D}(q)=\left\{q+w \in \mathbb{C}^{n} \mid \sum_{j=1}^{n} \frac{\left|z^{j}(w)\right|}{\tau_{j}(q)}<1\right\}
$$

is contained in $D$.

REMARK. In the previous lemma, with a slight abuse of notation we wrote $z^{j}(w)$ instead of $d\left(z^{j}\right)_{q}(w)$, where $w$ should be considered as a tangent vector to $\mathbb{C}^{n}$ in $q$. Being the $z^{j}$ 's affine maps, this amounts to considering $q$ as the origin. We shall use this convention from now on.

ProOF. Take $v \in \mathbb{C}^{n}$, with $\|v\|=1$; we want to estimate $\delta(q ; v)$. Set

$$
t=\sum_{j=1}^{n} \frac{\left|z^{j}(v)\right|}{\tau_{j}(q)} \text { and } \quad \alpha_{j}=\frac{\left|z^{j}(v)\right|}{t \tau_{j}(q)} \text { for } j=1, \ldots, n .
$$

Clearly, $\sum_{j} \alpha_{j}=1$.

Let $\gamma_{1}, \ldots, \gamma_{n}$ be the orthogonal unit vectors determined by the coordinate directions associated to $q$, so that $p_{j}=q+\tau_{j}(q) \gamma_{j}$ for $j=1, \ldots, n$. Every vector in the complex line $\mathbb{C} v$ is a positive real multiple of a vector of the form

$$
w=e^{i \theta} v=e^{i \theta} \sum_{j=1}^{n} z^{j}(v) \gamma_{j}=\sum_{j=1}^{n}\left|z^{j}(v)\right| \tilde{\gamma}_{j}
$$

for a suitable $\theta \in \mathbb{R}$, where $\tilde{\gamma}_{j}=e^{i \theta}\left(z^{j}(v) /\left|z^{j}(v)\right|\right) \gamma_{j}$. Since $p_{j}$ realizes the minimum distance from $q$ to the boundary of $D$ along the directions spanned by the vectors $\gamma_{j}, \ldots, \gamma_{n}$, the point $\tilde{p}_{j}=q+\tau_{j}(q) \tilde{\gamma}_{j}$ belongs to $\bar{D}$ for $j=1, \ldots, n$. The convexity of $D$ then implies that $q+w / t=\sum_{j=1}^{n} \alpha_{j} \tilde{p}_{j} \in \bar{D}$. Since this holds for any $\theta \in \mathbb{R}$, the euclidean distance from $q$ to $\partial D$ along the direction $v$ is at least $1 / t$, and thus

$$
\frac{1}{\delta(q ; v)} \leq t=\sum_{j=1}^{n} \frac{\left|z^{j}(v)\right|}{\tau_{j}(q)}
$$

Now let $w \in \mathbb{C}^{n}, w \neq 0$, be such that $\sum_{j=1}^{n}\left|z^{j}(w)\right| / \tau_{j}(q)<1$, and put $v=w /\|w\|$. Since $z^{j}(w)=\|w\| z^{j}(v)$ for $j=1, \ldots, n$, it follows that

$$
\|w\|<\left(\sum_{j=1}^{n} \frac{\left|z^{j}(v)\right|}{\tau_{j}(q)}\right)^{-1} \leq \delta(q ; v),
$$

and thus $q+w \in D$. 
Then

Proposition 1.6. Let $D \subset \mathbb{C}^{n}$ be a bounded convex domain. Let $x \in \partial D$ such that $\partial D$ is smooth near $x$, and assume that the type of $x$ is finite. Then there is a neighbourhood $U$ of $x$ such that for every $q \in D \cap U$ and $v \in \mathbb{C}^{n}$ we have

$$
\kappa_{D}(q ; v) \approx \sum_{j=1}^{n} \frac{\left|z^{j}(v)\right|}{\tau_{j}(q)},
$$

where the constants depend only on $D$.

PROOF. Lemma 1.5 yields $\kappa_{D}(q ; v) \leq \kappa_{A_{D}(q)}(q ; v)=\sum_{j=1}^{n}\left|z^{j}(v)\right| / \tau_{j}(q)$, and one direction is done.

Now, we clearly have

$$
z^{i}\left(p_{j}\right)=\tau_{j} \delta_{j}^{i}
$$

where $\delta_{j}^{i}$ is Kronecker's delta.

For $j=1, \ldots, n$, the tangent plane to $\partial D$ at $p_{j}$ is given by $\operatorname{Re} \chi_{j}(z)=0$, where

$$
\chi_{j}(z)=\sum_{h=1}^{n} \frac{\partial r}{\partial z^{h}}\left(p_{j}\right)\left[z^{h}\left(p_{j}\right)-z^{h}(z)\right] .
$$

Notice that, by convexity, $\operatorname{Re} \chi_{j}(z)>0$ for all $z \in D$ and $j=1, \ldots, n$. In particular, $\chi_{j}(D) \subset H$, the right half-plane in $\mathbb{C}$. Furthermore, by (1.11) and the right-hand side of (1.7), we have

$$
\left|\chi_{j}(q)\right|=\left|\frac{\partial r}{\partial z^{j}}\left(p_{j}\right)\right| \tau_{j} \lesssim \delta(q)
$$

Now, (1.12) yields

$$
\kappa_{D}(q ; v) \geq \kappa_{H}\left(\chi_{j}(q) ; d \chi_{j}(v)\right)=\frac{\left|d \chi_{j}(v)\right|}{2\left|\operatorname{Re} \chi_{j}(q)\right|} \geq \frac{\left|d \chi_{j}(v)\right|}{2\left|\chi_{j}(q)\right|} \gtrsim \frac{\left|d \chi_{j}(v)\right|}{2|\delta(q)|}
$$

and (1.6) yields

$$
\left|d \chi_{j}(v)\right| \geq\left|\frac{\partial r}{\partial z^{j}}\left(p_{j}\right)\right|\left|z^{j}(v)\right|-\sum_{i=1}^{j-1}\left|\frac{\partial r}{\partial z^{i}}\left(p_{j}\right)\right|\left|z^{i}(v)\right| .
$$

Then, first using the left-hand side of (1.7) and then (1.8), we get

$$
\begin{aligned}
\frac{\left|z^{j}(v)\right|}{\tau_{j}} & \lesssim \frac{1}{\delta(q)}\left|\frac{\partial r}{\partial z^{j}}\left(p_{j}\right)\right|\left|z^{j}(v)\right| \leq \frac{\left|d \chi_{j}(v)\right|}{\delta(q)}+\frac{1}{\delta(q)} \sum_{i=1}^{j-1}\left|\frac{\partial r}{\partial z^{i}}\left(p_{j}\right)\right|\left|z^{i}(v)\right| \\
& \lesssim \frac{\left|d \chi_{j}(v)\right|}{\delta(q)}+\sum_{i=1}^{j-1} \frac{\left|z^{i}(v)\right|}{\tau_{i}} .
\end{aligned}
$$


From this and (1.13) and by the induction on $j$ we obtain that

$$
\frac{\left|z^{j}(v)\right|}{\tau_{j}} \lesssim \kappa_{D}(q ; v)
$$

for $j=1, \ldots, n$, and the assertion follows.

COROLlaRY 1.7. Let $D \subset \mathbb{C}^{n}$ be a bounded convex domain. Let $x \in \partial D$ such that $\partial D$ is smooth near $x$, and assume that the line type of $x$ is $L \geq 2$. Then there is a neighbourhood $U$ of $x$ such that for all $q \in D \cap U$ and $v \in \mathbb{C}^{n}$ we have

$$
\frac{\|v\|}{\delta(q)^{1 / L}} \lesssim \kappa_{D}(q ; v) \lesssim \frac{\|v\|}{\delta(q)}
$$

and

$$
\frac{\|v\|}{\delta(q ; v)} \lesssim \kappa_{D}(q ; v)
$$

where the constants depend only on $D$.

PROOF. The second inequality in (1.16) follows immediately from Lemma 1.2, and thus does not depend on the fact that $D$ is of finite line type. For the first inequality, recalling (1.9) we get

$$
\frac{\|v\|}{\delta(q)^{1 / L}} \lesssim \frac{1}{\delta(q)^{1 / L}} \sum_{j=1}^{n}\left|z^{j}(v)\right| \lesssim \sum_{j=1}^{n} \frac{\left|z^{j}(v)\right|}{\tau_{j}} \lesssim \kappa_{D}(q ; v),
$$

by the previous proposition. Finally, (1.17) follows from (1.10) and (1.15).

A final estimate:

PROPOSITION 1.8. Let $D \subset \subset \mathbb{C}^{n}$ be a bounded convex domain. Let $x \in \partial D$ be such that $\partial D$ is smooth near $x$, and assume that the line type of $x$ is $L \geq 2$. Then there is a neighbourhood $U$ of $x$ such that if $p, q$ are in $D \cap U$ with $0<k_{D}(p, q)<c$, then

$$
\frac{\|p-q\|}{\delta(q ; p-q)} \lesssim k_{D}(p, q)
$$

where the constant is independent of $p$ and $q$ (but it depends on $c$ ).

PROOF. We use McNeal coordinates centered at $q$. For $j=1, \ldots, n$ we have

$$
\begin{aligned}
k_{D}(p, q) & \geq k_{H}\left(\chi_{j}(p), \chi_{j}(q)\right)=\operatorname{atanh}\left|\frac{\chi_{j}(p)-\chi_{j}(q)}{\chi_{j}(p)+\chi_{j}(q)}\right| \\
& \geq \operatorname{atanh} \frac{\left|\chi_{j}(p)-\chi_{j}(q)\right|}{\left|\chi_{j}(p)-\chi_{j}(q)\right|+2\left|\chi_{j}(q)\right|}=\frac{1}{2} \log \left(1+\frac{\left|\chi_{j}(p)-\chi_{j}(q)\right|}{\left|\chi_{j}(q)\right|}\right) .
\end{aligned}
$$


Since $k_{D}(p, q)<c$, recalling $(1.12)$ we get

$$
\frac{\left|\chi_{j}(p)-\chi_{j}(q)\right|}{\delta(q)} \lesssim \frac{\left|\chi_{j}(p)-\chi_{j}(q)\right|}{\left|\chi_{j}(q)\right|} \leq e^{2 k_{D}(p, q)}-1 \leq \frac{e^{2 c}-1}{c} k_{D}(p, q) .
$$

Now (1.6) yields

$$
\left|\chi_{j}(p)-\chi_{j}(q)\right| \geq\left|\frac{\partial r}{\partial z^{j}}\left(p_{j}\right)\right|\left|z^{j}(p)\right|-\sum_{i=1}^{j-1}\left|\frac{\partial r}{\partial z^{i}}\left(p_{j}\right)\right|\left|z^{i}(p)\right| .
$$

Then, first using the left-hand side of (1.7) and then (1.8), we get

$$
\begin{aligned}
\frac{\left|z^{j}(p)\right|}{\tau_{j}(q)} & \lesssim \frac{1}{\delta(q)}\left|\frac{\partial r}{\partial z^{j}}\left(p_{j}\right)\right|\left|z^{j}(p)\right| \leq \frac{\left|\chi_{j}(p)-\chi_{j}(q)\right|}{\delta(q)}+\frac{1}{\delta(q)} \sum_{i=1}^{j-1}\left|\frac{\partial r}{\partial z^{i}}\left(p_{j}\right)\right|\left|z^{i}(p)\right| \\
& \lesssim \frac{\left|\chi_{j}(p)-\chi_{j}(q)\right|}{\delta(q)}+\sum_{i=1}^{j-1} \frac{\left|z^{i}(p)\right|}{\tau_{i}(q)}
\end{aligned}
$$

From this and (1.19) and by the induction on $j$ we obtain that $\left|z^{j}(p)\right| / \tau_{j}(q) \lesssim k_{D}(p, q)$ for $j=1, \ldots, n$, and (1.18) follows from (1.10) applied with $v=p-q$.

\section{Complex geodesics}

A main technical tool for our work is the notion of complex geodesic. A complex geodesic in a domain $D \subset \subset \mathbb{C}^{n}$ is a holomorphic map $\varphi: \Delta \rightarrow D$, where $\Delta$ is the unit disk in the complex plane, which is an isometry with respect to the Poincare distance $\omega$ on $\Delta$ and the Kobayashi distance on $D$.

The complex geodesics are particularly well-behaved in convex domains. First of all, Lempert and Royden-Wong (see $[3,17,19,20,25])$ proved that for every pair of distinct points $z, w$ in a convex domain $D$ (respectively, for every point $z \in D$ and non-zero tangent direction $v \in \mathbb{C}^{n}$ ) there is a complex geodesic passing through $z$ and $w$ (respectively, passing through $z$ and tangent to $v$ ). Furthermore, a complex geodesic in a convex domain is automatically an isometry between the Poincaré metric on $\Delta$ and the Kobayashi metric on $D$ (and, conversely, any such isometry is a complex geodesic); and if a holomorphic map $\varphi: \Delta \rightarrow D$ preserves the distance between two given points (or the length of one given non-zero tangent vector) then $\varphi$ automatically is a complex geodesic.

In this section we shall discuss existence and uniqueness of complex geodesics passing through an interior point $z \in D$ and a boundary point $x \in \partial D$ when $D$ is of finite type, generalizing results known (see $[2,9]$ ) for strongly convex domains.

First, we prove a lemma. 
LEMMA 2.1. Let $D \subset \subset \mathbb{C}^{n}$ be a bounded convex domain. If $\varphi \in \operatorname{Hol}(\Delta, D)$ is a complex geodesic in $D$ such that $\varphi(0)=z_{0}$, then

$$
\delta(\varphi(\zeta)) \approx 1-|\zeta|
$$

where the constants depend only on $D$ and $z_{0}$.

PROOF. It suffices to apply (1.4) and (1.1) to $k_{D}(\varphi(0), \varphi(\zeta))$, recalling that

$$
k_{D}(\varphi(0), \varphi(\zeta))=\omega(0, \zeta)=\frac{1}{2} \log \frac{1+|\zeta|}{1-|\zeta|}
$$

To prove the existence of a complex geodesic passing through a point in the boundary we first need to know that the complex geodesics at least extend continuously to the boundary. For that, the following criterion due to Hardy and Littlewood will be useful:

THEOREM $2.2([14])$. Let $f \in \operatorname{Hol}(\Delta, \mathbb{C})$ and fix $\alpha \in(0,1)$. Then $f$ belongs to the Hölder space $C^{0, \alpha}(\bar{\Delta})$ if and only $\left|f^{\prime}(\zeta)\right| \lesssim(1-|\zeta|)^{\alpha-1}$ for all $\zeta \in \Delta$.

Now we can prove that every complex geodesic in a convex domain of finite type extends continuously to $\partial \Delta$.

THEOREM 2.3. Let $D \subset \mathbb{C}^{n}$ be a bounded convex domain of finite type $L$ and let $\varphi: \Delta \rightarrow D$ be a complex geodesic. Then each component of $\varphi$ belongs to the Hölder space $C^{0,1 / L}(\bar{\Delta})$.

PROOF. By Lemma 1.1 and Corollary 1.7 (and recalling that a complex geodesic is automatically an isometry between the Poincaré metric and the Kobayashi metric) we get

$$
\frac{\left\|\varphi^{\prime}(\zeta)\right\|}{\delta(\varphi(\zeta))^{1 / L}} \lesssim \kappa_{D}\left(\varphi(\zeta) ; \varphi^{\prime}(\zeta)\right)=\kappa_{\Delta}(\zeta ; 1)=\frac{1}{1-|\zeta|^{2}}
$$

Hence Lemma 2.1 yields

$$
\left\|\varphi^{\prime}(\zeta)\right\| \lesssim \frac{1}{1-|\zeta|^{2}} \delta(\varphi(\zeta))^{1 / L} \lesssim\left(1-|\zeta|^{(1 / L)-1}\right.
$$

and the desired regularity of $\varphi$ follows from Theorem 2.2 .

As a consequence we can prove the following:

PROPOSITION 2.4. Let $D \subset \subset \mathbb{C}^{n}$ be a bounded convex domain of finite type $L$. Then for any $z_{0} \in D$ and $x \in \partial D$ there exists a complex geodesic $\varphi: \Delta \rightarrow D$ such that $\varphi(0)=z_{0}$ and $\varphi(1)=x$. 
ProOF. Let $\left\{z_{k}\right\} \subset D$ be a sequence converging to $x$. The convexity of $D$ yields for each $k \in \mathbb{N}$ a complex geodesic $\varphi_{k}: \Delta \rightarrow D$ such that $\varphi_{k}(0)=z_{0}$ and $\varphi_{k}\left(t_{k}\right)=z_{k}$ for a suitable $t_{k}>0$. Moreover $D$, being a bounded convex domain, is taut, and thus up to a subsequence we can assume that the sequence $\left\{\varphi_{k}\right\}$ converges to a map $\varphi \in \operatorname{Hol}(\Delta, D)$, and that $t_{k} \rightarrow 1^{-}$. Clearly, $\varphi(0)=z_{0}$ and for all $\zeta \in \Delta$,

$$
k_{D}\left(z_{0}, \varphi(\zeta)\right)=\lim _{k \rightarrow \infty} k_{D}\left(z_{0}, \varphi_{k}(\zeta)\right)=\omega(0, \zeta)
$$

As remarked before, this is enough to assure that $\varphi$ is a complex geodesic. By Theorem 2.3 , it belongs to $C^{0.1 / L}(\bar{\Delta})$, and then we clearly have $\varphi(1)=x$.

Our next goal is to prove (under suitable hypotheses) the uniqueness of the complex geodesic passing through a given point $z_{0} \in D$ and a given point $x \in \partial D$. To do so we need to describe in more detail the theory surrounding complex geodesics in convex domains.

Let $D \subset \subset \mathbb{C}^{n}$ be a bounded convex domain, and $\varphi: \Delta \rightarrow D$ a complex geodesic. Lempert and Royden-Wong proved the existence of a holomorphic dual map $\varphi^{*}: \Delta \rightarrow$ $\mathbb{C}^{n}$ satisfying the following properties:

(a) the components of $\varphi^{*}$ belongs to the Hardy space $H^{1}(\Delta)$;

(b) $\varphi^{*}$ is uniquely determined up to a constant positive multiple as soon as there exists a unique supporting hyperplane at every point of $\partial D$ (for example, if $\partial D$ is $C^{2}$ );

(c) for almost all $\tau \in \partial \Delta$ we have $\varphi^{*}(\tau)=\tau \mu(\tau) \overline{\mathbf{n}_{\varphi(\tau)}}$ for a unique (up to a positive multiple) function $\mu \in L^{1}(\partial \Delta)$;

(d) $\varphi^{*}$ is never vanishing in $\Delta$ and for every $z \in D$ there exists a unique $\zeta=\tilde{p}(z) \epsilon$ $\Delta$ such that $\left\langle z-\varphi(\zeta), \varphi^{*}(\zeta)\right\rangle=0$, where $\langle z, w\rangle=(z, \bar{w})$;

(e) $\tilde{p}: D \rightarrow \Delta$, the left inverse of $\varphi$, is holomorphic, $\tilde{p} \circ \varphi=\mathrm{id}_{\Delta}$, and $p=\varphi \circ \tilde{p}$ is a holomorphic retraction of $D$ onto $\varphi(\Delta)$;

(f) $\operatorname{Re}\left\langle\varphi^{\prime}(0), \varphi^{*}(0)\right\rangle>0$.

We now need to recover in the finite type case some properties of the dual map $\varphi^{*}$ and of the associated maps $p$ and $\tilde{p}$ already known in the strongly convex setting.

LEMMA 2.5. Let $D \subset \subset \mathbb{C}^{n}$ be a bounded convex domain of finite type, and let $\varphi: \Delta \rightarrow D$ be a complex geodesic. Set, for almost all $\tau \in \partial \Delta, v(\tau)=\left(\varphi^{\prime}(\tau), \mathbf{n}_{\varphi(\tau)}\right)$. Then $v, 1 / v \in L^{\infty}(\partial \Delta)$.

PROOF. Take $\tau \in \partial \Delta$. By (1.5), (2.1) and since $\kappa_{\Delta}(t \tau ; 1)=1 /\left(1-t^{2}\right)$ we have

$$
\left|\left(\varphi^{\prime}(t \tau), \mathbf{n}_{\varphi(t \tau)}\right)\right| \lesssim \delta(\varphi(t \tau)) \kappa_{D}\left(\varphi(t \tau) ; \varphi^{\prime}(t \tau)\right) \lesssim(1-t) \kappa_{\Delta}(t \tau ; 1) \leq 1 .
$$

Letting $t$ go to 1 , we obtain that $v \in L^{\infty}(\partial \Delta)$. 
Now, applying (1.3) to $z=\varphi(t \tau)$ and $v=\varphi^{\prime}(t \tau)$, we get

$$
\left|\left(\varphi^{\prime}(t \tau), \mathbf{n}_{\varphi(t \tau)}\right)\right|=\left\|v_{N}\right\| \gtrsim \kappa_{D}\left(\varphi(t \tau) ; \varphi^{\prime}(t \tau)\right) \delta(\varphi(t \tau))-\left\|v_{T}\right\| \delta(\varphi(t \tau))^{1 / 2} .
$$

So, by $(2.1)$,

$$
\left|\left(\varphi^{\prime}(t \tau), \mathbf{n}_{\varphi(t \tau)}\right)\right| \gtrsim(1-t) \kappa_{\Delta}(t \tau ; 1)-\left\|v_{T}\right\|(1-t)^{1 / 2} \geq 1 / 2-\left\|v_{T}\right\|(1-t)^{1 / 2} .
$$

Letting again $t$ go to 1 , we find that $1 / v \in L^{\infty}(\partial \Delta)$.

Now, the curve $\theta \mapsto \varphi\left(e^{i \theta}\right)$ is almost everywhere differentiable, and its tangent at $\varphi\left(e^{i \theta}\right)$ is orthogonal to $\mathbf{n}_{\varphi\left(e^{i \theta}\right)}$. From this it is easy to prove (see [3, page 330] or [20]) that $\operatorname{Im}\left\langle\varphi^{\prime}\left(e^{i \theta}\right), \varphi^{*}\left(e^{i \theta}\right)\right\rangle=0$ for almost every $\theta$, and thus $\operatorname{Im}\left\langle\varphi^{\prime}, \varphi^{*}\right\rangle \equiv 0$ on $\Delta$. Since Lemma 2.5 yields $\left\langle\varphi^{\prime}, \varphi^{*}\right\rangle \in L^{1}(\Delta)$, it follows that $\left\langle\varphi^{\prime}, \varphi^{*}\right\rangle$ is a positive constant. Now, $\varphi^{*}$ is defined up to a positive multiple; therefore we can (and we shall) always assume

$$
\left\langle\varphi^{\prime}, \varphi^{*}\right\rangle \equiv 1
$$

In particular, the curve $t \mapsto \varphi(t)$ approaches $\varphi(1)$ non-tangentially.

COROLLARY 2.6. Let $D \subset \subset \mathbb{C}^{n}$ be a bounded convex domain of finite type $L$, and let $\varphi: \Delta \rightarrow D$ be a complex geodesic. Then:

(i) all the components of $\varphi^{*}$ belong to the Hölder class $C^{0,1 / L}(\bar{\Delta})$;

(ii) the function $v(\tau)=\left(\varphi^{\prime}(\tau), \mathbf{n}_{\varphi(\tau)}\right)$ belongs to the Hölder class $C^{0,1 / L}(\partial \Delta)$;

(iii) the function $\tilde{p}$ belongs to the class $C^{1}(\bar{D})$.

ProOF. Setting $\mu(\tau)=\left\|\varphi^{*}(\tau)\right\|$, by (2.2) we get $\tau \mu(\tau) v(\tau)=1$ almost everywhere on $\partial \Delta$; since $1 / v \in L^{\infty}(\partial \Delta)$, this implies that $v$ belongs to the same Hölder class as $\mu$ (if any). Furthermore, since we know that $\varphi \in C^{0,1 / L}(\bar{\Delta})$, a classical result by Hardy and Littlewood [14] says that $\varphi^{*}$ is $1 / L$-Hölder if and only if $\mu$ is. Given this, since $\varphi^{*}$ and $\varphi$ extends continuously to the boundary, so does $\tilde{p}$; and the regularity of $d \tilde{p}$ is the same as the regularity of $\varphi^{*}$, that is $\tilde{p} \in C^{1}(\bar{D})$.

So it suffices to prove that $\mu \in C^{0,1 / L}(\partial \Delta)$, and this can be achieved as in [3, Theorem 2.6.34] or [20].

In particular, for every $z \in D$ and $v \in \mathbb{C}^{n}$ we have $d \tilde{p}_{z}(v)=\left\langle v, \varphi^{*}(\tilde{p}(z))\right\rangle$, and for every $\tau \in \partial \Delta$ and $v \in \mathbb{C}^{n}$ we have

$$
d \tilde{p}_{\varphi(\tau)}(v)=\left\langle v, \varphi^{*}(\tau)\right\rangle=\frac{\left(v, \mathbf{n}_{\varphi(\tau)}\right)}{\left(\varphi^{\prime}(\tau), \mathbf{n}_{\varphi(\tau)}\right)} .
$$

We shall say that a convex domain $D$ is strictly linearly convex if for every $x \in \partial D$ the complex tangent hyperplane $T_{x}^{\mathrm{C}}(\partial D)=T_{x}(\partial D) \cap i T_{x}(\partial D)$ intersects $\partial D$ only 
in $x$. Strongly convex domains and convex domains with real-analytic boundary (which are automatically of finite type) are necessarily strictly linearly convex, but the converse is not true.

Our interest in this notion lies in the following

LEMMA 2.7. Let $D \subset \subset \mathbb{C}^{n}$ be a bounded convex domain of finite type, $\varphi: \Delta \rightarrow D$ a complex geodesic, and $\tilde{p}: \bar{D} \rightarrow \bar{\Delta}$ its left inverse. Then if $z \in \bar{D}$ does not belong to any hyperplane $T_{\varphi(\tau)}^{\mathrm{C}} \partial D$ for all $\tau \in \partial \Delta$, then $\tilde{p}(z) \in \Delta$.

PROOF. The number of solutions in $\Delta$ of $\left\langle z-\varphi(\zeta), \varphi^{*}(\zeta)\right\rangle=0$ is equal to the winding number of the function $g_{z}(\zeta)=\left\langle z-\varphi(\zeta), \varphi^{*}(\zeta)\right\rangle$. Arguing as in [3, Proposition 2.6.22] one sees that for $z \in \bar{D}$ the winding number is 1 unless $g_{2}(\tau)=0$ for some $\tau \in \partial \Delta$, which is equivalent to having $z \in T_{\varphi}^{\mathrm{C}}(\tau) \partial D$ for some $\tau \in \partial \Delta$ (and in that case $\tilde{p}(z)=\varphi(\tau) \in \partial D)$.

We are finally ready to prove the uniqueness of complex geodesics:

THEOREM 2.8. Let $D \subset \subset \mathbb{C}^{n}$ be a bounded strictly linearly convex domain of finite type. Then

(i) for any pair of distinct points $z_{0}, w_{0} \in D$ there is a unique complex geodesic $\varphi: \Delta \rightarrow D$ such that $\varphi(0)=z_{0}$ and $\varphi(t)=w_{0}$, where $t=\tanh k_{D}\left(z_{0}, w_{0}\right)$;

(ii) for any $z_{0} \in D$ and $x \in \partial D$ there exists a unique complex geodesic $\varphi$ such that $\varphi(0)=z_{0}$ and $\varphi(1)=x$.

PROOF. (i) We shall not need this fact in the sequel, but it is interesting to compare its proof with the proof of (ii). Assume, by contradiction, that there are two complex geodesics $\varphi$ and $\psi$ passing through $z_{0}$ and $w_{0}$ as required, and let $\tilde{p}$ be the left inverse of $\varphi$. Then $\tilde{p} \circ \psi$ is a holomorphic self-map of the disk with two fixed points, and hence $\tilde{p} \circ \psi=\mathrm{id}_{\Delta}$. The previous lemma then implies $\left.\left.\varphi\right|_{\partial \Delta} \equiv \psi\right|_{\partial \Delta}$, and thus $\varphi \equiv \psi$.

(ii) Assume now that $\varphi$ and $\psi$ are two complex geodesics with $\varphi(0)=\psi(0)=z_{0}$ and $\varphi(1)=\psi(1)=x$. Denote by $\tilde{p}$ the left inverse of $\varphi$, and by $\tilde{q}$ the left inverse of $\psi$. Set $f=\tilde{p} \circ \psi$ and $g=\tilde{q} \circ \varphi$. Clearly, $f, g \in \operatorname{Hol}(\Delta, \Delta) \cap C(\bar{\Delta}), f(0)=g(0)=0$ and $f(1)=g(1)=1$. Again, it suffices to prove that $f=\mathrm{id}_{\Delta}$.

First, we prove that there exists the radial limit of $f^{\prime}$ at 1 . By the classical JuliaWolff-Carathéodory theorem, it is enough to show that

$$
\liminf _{t \rightarrow 1}[\omega(0, t)-\omega(0, f(t))]<\infty
$$

Now, $\varphi(t)$ and $\psi(t)$ are non-tangential $x$-curves; therefore there is $M>1$ such that 
for all $t$ close enough to 1 we have

$$
\max \left\{\frac{\|\varphi(t)-x\|}{\delta(\varphi(t))}, \frac{\|\psi(t)-x\|}{\delta(\psi(t))}\right\}<M .
$$

Moreover, by $(2.1), \delta(\varphi(\zeta)) \approx \delta(\psi(\zeta)) \approx(1-|\zeta|)$, and therefore Lemma 1.1 (ii) yields

$$
\begin{aligned}
\omega(0, t) & -\omega(0, f(t)) \\
= & k_{D}\left(z_{0}, \varphi(t)\right)-k_{D}\left(z_{0}, p \circ \psi(t)\right) \leq k_{D}(\varphi(t), p \circ \psi(t)) \leq k_{D}(\varphi(t), \psi(t)) \\
\leq & \frac{1}{2} \log \left(1+\frac{\|\varphi(t)-\psi(t)\|}{\delta(\varphi(t))}\right)+\frac{1}{2} \log \left(1+\frac{\|\varphi(t)-\psi(t)\|}{\delta(\psi(t))}\right)+c \\
\leq & \frac{1}{2} \frac{\|\varphi(t)-\psi(t)\|}{\delta(\varphi(t))}+\frac{1}{2} \frac{\|\varphi(t)-\psi(t)\|}{\delta(\psi(t))}+c \\
\leq & \frac{1}{2}\left(\|\varphi(t)-x\|+\|\psi(t)-x\|\left(\frac{1}{\delta(\varphi(t))}+\frac{1}{\delta(\psi(t))}\right)+c\right. \\
\leq & \frac{\|\varphi(t)-x\|}{\delta(\varphi(t))}+\frac{\|\psi(t)-x\|}{\delta(\psi(t))}+c \lesssim 2 M+c .
\end{aligned}
$$

Hence there exist the radial limits $f^{\prime}(1)$ and $g^{\prime}(1)$. Since $f(0)=g(0)=0$, a classical result (see [3, Corollary 1.2.10]) says that $f^{\prime}(1) \geq 1$ and $f^{\prime}(1)=1$ if and only if $f$ is the identity (and likewise for $g$ ). But then (2.3) and the continuity of $\left(\psi^{\prime}, \mathbf{n}_{\psi}\right)$ and $\left(\varphi^{\prime}, \mathbf{n}_{\varphi}\right)$ yields $1 \leq f^{\prime}(1)=\left(\psi^{\prime}(1), \mathbf{n}_{x}\right) /\left(\varphi^{\prime}(1), \mathbf{n}_{x}\right)$. Analogously,

$$
1 \leq g^{\prime}(1)=\frac{\left(\varphi^{\prime}(1), \mathbf{n}_{x}\right)}{\left(\psi^{\prime}(1), \mathbf{n}_{x}\right)}=\frac{1}{f^{\prime}(1)}
$$

therefore $f^{\prime}(1)=g^{\prime}(1)=1$, and thus $f=\mathrm{id}_{\Delta}$, as claimed.

REMARK. We do not actually need the full power of strict linear convexity to get uniqueness of complex geodesics; we can allow some intersection between complex tangent hyperplanes and the boundary. Indeed, let $E \subset \partial D$ be the set of points $x \in \partial D$ such that $T_{x}^{\mathrm{C}}(\partial D) \cap \partial D$ contains at least two points (and thus, by convexity, a real segment). Then the previous proof shows that if $\varphi$ is a complex geodesic such that $\varphi(\partial \Delta) \cap E$ is not of full measure then $\varphi$ is the unique complex geodesic passing through $\varphi(0)$ and any other point in its image. Since the tangent vector to $\varphi(\partial \Delta)$, which exists a.e., is transversal to the complex tangent hyperplanes, a sufficient condition for this is that the set $E$ has zero 2-dimensional Hausdorff measure.

We shall not even need the full uniqueness statement. What is really needed in the sequel is the following 
COROLlARY 2.9. Let $D \subset \subset \mathbb{C}^{n}$ be a bounded strictly linearly convex domain of finite type, fix $z_{0} \in D$ and denote for every $z \in \bar{D}$ by $\varphi_{z}$ the only complex geodesic with $\varphi_{z}(0)=z_{0}$ and $\varphi_{z}\left(t_{0}\right)=z$, where $t_{0}=\tanh k_{D}\left(z_{0}, z\right) \in[0,1]$. Then $z \mapsto \varphi_{z}$ is continuous.

PROOF. Let $\left\{z_{k}\right\} \subset \bar{D}$ be a sequence converging to $z \in \bar{D}$. Then every subsequential limit of $\left\{\varphi_{z_{k}}\right\}$ is a complex geodesic passing through $z_{0}$ and $z$, and thus (by uniqueness) it is $\varphi_{z}$. By tautness, this imply that $\varphi_{z}$ is the limit of $\left\{\varphi_{z_{k}}\right\}$, as claimed.

More generally, a convex domain $D$ is solid in $z_{0} \in D$ if for every $z \in \bar{D}$ it is possible to choose a complex geodesic $\varphi_{z}$ with $\varphi_{z}(0)=z_{0}$ and $\varphi_{z}\left(t_{0}\right)=z$, where $t_{0}=\tanh k_{D}\left(z_{0}, z\right)$, in such a way that the map $z \mapsto \varphi_{z}$ is continuous. We have just proved that strictly linearly convex domains of finite type (or, more generally, convex domains of finite type strictly linearly convex except in a set of zero 2-dimensional Hausdorff measure) are solid in every point. Another example is given by convex circular domains (no regularity assumption on the boundary) which are always solid with respect to the origin (it suffices to choose $\varphi_{z}(\zeta)=\zeta x$, where $x$ is the intersection of $\partial D$ with the real half-line issuing from the origin and passing through $z$; see [3, Corollary 2.6.4]).

In Section 4 we shall prove a Julia-Wolff-Carathéodory theorem for convex domains $D$ of finite type solid in a given point $z_{0} \in D$. Actually, the solid assumption will be needed only for the following two lemmas.

LEMMA 2.10. Let $D \subset \subset \mathbb{C}^{n}$ be a bounded convex domain solid in $z_{0} \in D$. Then for every $x \in \partial D$ and $z \in D$ we have

$$
\underset{w \rightarrow x}{\limsup }\left[k_{D}(z, w)-k_{D}\left(z_{0}, w\right)\right]=\lim _{t \rightarrow 1^{-}}\left[k_{D}\left(z, \varphi_{x}(t)\right)-\omega(0, t)\right] .
$$

PROOF. First of all, it is easy to check that the function $t \mapsto k_{D}\left(z, \varphi_{x}(t)\right)-\omega(0, t)$ is not increasing for $t \in[0,1)$; let $h_{z}(x)$ denote its limit as $t \rightarrow 1^{-}$. By definition $h_{z}(x) \leq \lim \sup _{w \rightarrow x}\left[k_{D}(z, w)-k_{D}(0, w)\right]$. To prove the converse inequality we need to show that for every $\varepsilon>0$ there is $\delta>0$ so that if $\|w-x\|<\delta$ then

$$
k_{D}(z, w)-k_{D}(0, w)<h_{z}(x)+\varepsilon
$$

Choose $t_{0}<1$ so that $k_{D}\left(z, \varphi_{x}\left(t_{0}\right)\right)-\omega\left(0, t_{0}\right) \leq h_{z}(x)+\varepsilon / 2$, and then fix $\delta>0$ such that if $\|w-x\|<\delta$ then $k_{D}\left(z_{0}, w\right)>\omega\left(0, t_{0}\right)$ and

$$
\left|k_{D}\left(z, \varphi_{w}\left(t_{0}\right)\right)-k_{D}\left(z, \varphi_{x}\left(t_{0}\right)\right)\right|<\varepsilon / 2
$$


such a $\delta$ exists because $D$ is solid in $z_{0}$. Then

$$
\begin{aligned}
k_{D}(z, w)-k_{D}\left(z_{0}, w\right) & =k_{D}\left(z, \varphi_{w}\left(\tanh k_{D}\left(z_{0}, w\right)\right)\right)-\omega\left(0, \tanh k_{D}\left(z_{0}, w\right)\right) \\
& \leq k_{D}\left(z, \varphi_{w}\left(t_{0}\right)\right)-\omega\left(0, t_{0}\right) \\
& \leq k_{D}\left(z, \varphi_{x}\left(t_{0}\right)\right)-\omega\left(0, t_{0}\right)+\varepsilon / 2 \leq h_{z}(x)+\varepsilon
\end{aligned}
$$

and we are done.

LEMMA 2.11. Let $D \subset \subset \mathbb{C}^{n}$ be a convex domain solid in $z_{0} \in D$. Then for all $x \in \partial D$ and $f \in \operatorname{Hol}(D, \Delta)$ we have

$$
\liminf _{z \rightarrow x}\left[k_{D}\left(z_{0}, z\right)-\omega(0, f(z))\right]=\lim _{t \rightarrow 1^{-}}\left[\omega(0, t)-\omega\left(0, f\left(\varphi_{x}(t)\right)\right)\right] .
$$

PROOF. First of all, it is easy to check that the function $t \mapsto \omega(0, t)-\omega\left(0, f\left(\varphi_{x}(t)\right)\right)$ is not decreasing in $[0,1)$; let $h \in \mathbb{R} \cup\{+\infty\}$ denote its limit as $t \rightarrow 1^{-}$. Clearly,

$$
\liminf _{z \rightarrow x}\left[k_{D}\left(z_{0}, z\right)-\omega(0, f(z))\right] \leq h .
$$

To prove the converse inequality we need to show that for every $\varepsilon>0$ there is a $\delta>0$ so that $\|z-x\|<\delta$ implies $k_{D}\left(z_{0}, z\right)-\omega(0, f(z)) \geq h-\varepsilon$. Choose $t_{0}>0$ so that $\omega\left(0, t_{0}\right)-\omega\left(0, f\left(\varphi_{x}\left(t_{0}\right)\right)\right) \geq h-\varepsilon / 2$, and take $\delta>0$ so that if $z \in D$ is such that $\|z-x\|<\delta$ then $k_{D}\left(z_{0}, z\right)>\omega\left(0, t_{0}\right)$ and $\left|\omega\left(0, f\left(\varphi_{x}\left(t_{0}\right)\right)\right)-\omega\left(0, f\left(\varphi_{z}\left(t_{0}\right)\right)\right)\right|<\varepsilon / 2$. In particular,

$$
\begin{aligned}
\omega(0, t)-\omega\left(0, f\left(\varphi_{z}(t)\right)\right) & \geq \omega\left(0, t_{0}\right)-\omega\left(0, f\left(\varphi_{z}\left(t_{0}\right)\right)\right) \\
& \geq \omega\left(0, t_{0}\right)-\omega\left(0, f\left(\varphi_{x}\left(t_{0}\right)\right)\right)-\varepsilon / 2 \geq h-\varepsilon
\end{aligned}
$$

for all $t \geq t_{0}$. Then

$$
\begin{aligned}
k_{D}\left(z_{0}, z\right)-\omega(0, f(z)) & =\omega\left(0, \tanh k_{D}\left(z_{0}, z\right)\right)-\omega\left(0, f\left(\varphi_{z}\left(\tanh k_{D}\left(z_{0}, z\right)\right)\right)\right) \\
& \geq h-\varepsilon
\end{aligned}
$$

and we are done.

\section{The Lindelöf principle}

As described in the introduction, to prove the Julia-Wolff-Caratheodory theorem one needs the Lindelöf principle. The aim of this section is to prove the Lindelöf principle for a convex domain of finite type.

Let $D \subset \subset \mathbb{C}^{n}$ be a bounded convex domain; in this section we shall fix once for all: a point $z_{0} \in D$; a point $x \in \partial D$ such that $\partial D$ is of finite type in a neighbourhood of $x$; 
and a complex geodesic $\varphi_{x}$ such that $\varphi_{x}(0)=z_{0}$ and $\varphi_{x}(1)=x$, with corresponding left-inverse $\tilde{p}_{x}$ and holomorphic retraction $p_{x}=\varphi_{x} \circ \tilde{p}_{x}$. Except in a secondary lemma, we shall not assume that $D$ is solid in $z_{0}$; in particular, we shall not assume that $D$ is strictly linearly convex.

Our previous approaches to Lindelöf principles (see [4-6] and [7]) were based on the (small) $K$-regions of vertex $x \in \partial D$, amplitude $M>1$ and pole $z_{0} \in D$, given by

$$
K_{z_{0}}(x, M)=\left\{z \in D \mid \limsup _{w \rightarrow x}\left[k_{D}(z, w)-k_{D}\left(z_{0}, w\right)\right]+k_{D}\left(z_{0}, z\right)<\log M\right\} .
$$

(Caution: in $[3,4]$ this region was denoted by $H_{2_{0}}(x, M)$ because there we also needed another approach region defined using the lim inf instead of the $\lim$ sup.) When $D=\Delta$ the $K$-regions of pole the origin are the classical Stolz regions

$$
K_{\Delta}(\tau, M)=\left\{\zeta \in \Delta \mid \frac{|\tau-\zeta|}{1-|\zeta|}<M\right\} .
$$

Later on, we shall also need the (small) horosphere of center $x \in \partial D$, radius $R>0$ and pole $z_{0} \in D$ :

$$
E_{z_{0}}(x, M)=\left\{z \in D \mid \limsup _{w \rightarrow x}\left[k_{D}(z, w)-k_{D}\left(z_{0}, w\right)\right]<(1 / 2) \log R\right\} .
$$

In the unit disk small horospheres of pole the origin are the classical horocycles

$$
E_{\Delta}(\tau, R)=\left\{\zeta \in \Delta \mid \frac{|\tau-\zeta|^{2}}{1-|\zeta|^{2}}<R\right\} .
$$

It turns out that in convex domains the choice of a different kind of approach regions yields better results. A $T$-region of vertex $x \in \partial D$, amplitude $M>1$, pole $z_{0} \in D$ and girth $0<\delta<1$ is

$$
T_{z_{0}}(x, M, \delta)=\left\{z \in D \mid \tilde{p}_{x}(z) \in K_{\Delta}(1, M), k_{D}\left(z, p_{x}(z)\right)<\omega(0, \delta)\right\} .
$$

If $D$ is solid in $z_{0}$, the $T$-regions are actually smaller than $K$-regions (but we stress that we shall not need this result in the sequel):

LEMMA 3.1. Let $D \subset \subset \mathbb{C}^{n}$ be a bounded convex domain solid in $z_{0} \in D$. Then for all $x \in \partial D, M>1$ and $0<\delta<1$ we have $T_{z_{0}}(x, M, \delta) \subset K_{z_{0}}(x, M(1+\delta) /(1-\delta))$.

Proof. In fact, take $z \in T_{z_{0}}(x, M, \delta)$ and set $\zeta=\tilde{p}_{x}(z)$. Then we have

$$
\begin{aligned}
k_{D}(z, w)-k_{D}\left(z_{0}, w\right)+k_{D}\left(z_{0}, z\right) \\
\quad \leq 2 k_{D}\left(z, p_{x}(z)\right)+k_{D}\left(p_{x}(z), w\right)-k_{D}\left(z_{0}, w\right)+k_{D}\left(z_{0}, p_{x}(z)\right) \\
\quad<2 \omega(0, \delta)+k_{D}\left(p_{x}(z), w\right)-k_{D}\left(z_{0}, w\right)+k_{D}\left(z_{0}, p_{x}(z)\right)
\end{aligned}
$$


for all $w \in D$. Now, recalling Lemma 2.10 we find

$$
\begin{aligned}
\limsup _{w \rightarrow x} & {\left[k_{D}\left(p_{x}(z), w\right)-k_{D}\left(z_{0}, w\right)\right]+k_{D}\left(z_{0}, p_{x}(z)\right) } \\
& =\lim _{t \rightarrow 1^{-}}\left[k_{D}\left(p_{x}(z), \varphi_{x}(t)\right)-k_{D}\left(z_{0}, \varphi_{x}(t)\right)\right]+k_{D}\left(z_{0}, p_{x}(z)\right) \\
& =\lim _{t \rightarrow 1^{-}}\left[\omega\left(\tilde{p}_{x}(z), t\right)-\omega(0, t)\right]+\omega\left(0, \tilde{p}_{x}(z)\right)<\log M,
\end{aligned}
$$

because $\tilde{p}_{x}(z) \in K_{\Delta}(1, M)$. Therefore

$$
\underset{w \rightarrow x}{\limsup }\left[k_{D}(z, w)-k_{D}\left(z_{0}, w\right)\right]+k_{D}\left(z_{0}, z\right)<\log \left(\frac{1+\delta}{1-\delta}\right)+\log M,
$$

that is $z \in K_{z_{0}}(x, M(1+\delta) /(1-\delta))$ as claimed.

To state our new Lindelöf principle we recall a few customary definitions. An $x$-curve is a continuous curve $\gamma:[0,1) \rightarrow D$ such that $\gamma(t) \rightarrow x$ as $t \rightarrow 1^{-}$. For every $x$-curve $\gamma$ in $D$ we set $\gamma_{x}=p_{x} \circ \gamma$ and $\tilde{\gamma}_{x}=\tilde{p}_{x} \circ \gamma$; the latter is a 1-curve in $\Delta$. We shall say that an $x$-curve $\gamma$ is special if $k_{D}\left(\gamma(t), \gamma_{x}(t)\right) \rightarrow 0$ as $t \rightarrow 1^{-}$, and that it is $M$-restricted if $\tilde{\gamma}_{x}(t) \in K_{\Delta}(1, M)$ eventually; in particular, if $\gamma$ is restricted then $\tilde{\gamma}_{x}$ approaches 1 non-tangentially. Notice that if $\gamma$ is a special $M$-restricted $x$-curve then for all $0<\delta<1$ we have $\gamma(t) \in T_{z_{0}}(x, M, \delta)$ eventually.

We shall say that a map $f: D \rightarrow \mathbb{C}$ is $K$-bounded at $x \in \partial D$ if it is bounded in every $K$-region of vertex $x$ (with the bound depending on the amplitude of the $K$-region). On the other hand, we shall say that a map $f: D \rightarrow \mathbb{C}$ is $T$-bounded at $x \in \partial D$ if there exists $0<\delta_{0}<1$ such that $f$ is bounded in every $T$-region $T_{z_{0}}\left(x, M, \delta_{0}\right)$ of vertex $x$ and girth $\delta_{0}$, (with the bound depending again on the amplitude $M$ ). Note that, by Lemma 3.1, if $D$ is solid in $z_{0}$ then if a function $f$ is $K$-bounded at $x$ then it is $T$-bounded at $x$.

Finally, we shall say that the map $f$ has $K$-limit $L \in \mathbb{C}$ at $x \in \partial D$ if $f(z) \rightarrow L$ when $z \rightarrow x$ staying inside any $K$-region $K_{z_{0}}(x, M)$; and that it has restricted $K$-limit $L \in \mathbb{C}$ at $x$ if $f(\gamma(t)) \rightarrow L$ as $t \rightarrow 1^{-}$for any special restricted $x$-curve $\gamma$. Then our new Lindelöf principle is:

THEOREM 3.2. Let $D \subset \mathbb{C}^{n}$ be a bounded convex domain, and fix $z_{0} \in D$. Let $x \in \partial D$ such that $\partial D$ is smooth near $x$, and assume that the line type of $x$ is finite. Choose a complex geodesic $\varphi_{x}$ such that $\varphi_{x}(0)=z_{0}$ and $\varphi_{x}(1)=x$, and let $f \in \operatorname{Hol}(D, \mathbb{C})$ be $T$-bounded at $x \in \partial D$. If $f\left(\gamma^{o}(t)\right) \rightarrow L$ as $t \rightarrow 1^{-}$for a special restricted $x$-curve $\gamma^{\circ}$, then $f$ has restricted $K$-limit $L$ at $x$.

Proof. Let $\gamma$ be a special $M$-restricted $x$-curve. For $t \in[0,1)$ let the map $\Psi_{t} \in \operatorname{Hol}\left(\mathbb{C}, \mathbb{C}^{n}\right)$ be defined by setting $\Psi_{t}(\zeta)=\gamma_{x}(t)+\zeta\left(\gamma(t)-\gamma_{x}(t)\right)$. First of all 
note that for any $R>1$ there is $0<t_{R}<1$ such that $\Psi_{t}\left(\Delta_{R}\right) \subset D$ for all $t \geq t_{R}$, where $\Delta_{R}=R \Delta$. In fact, $\Psi_{t}\left(\Delta_{R}\right) \subset D$ if and only if for all $\zeta \in \Delta_{R}$ we have

$$
\left\|\Psi_{t}(\zeta)-\gamma_{x}(t)\right\|=|\zeta|\left\|\gamma(t)-\gamma_{x}(t)\right\|<\delta\left(\gamma_{x}(t) ; \gamma(t)-\gamma_{x}(t)\right),
$$

that is

$$
\frac{\left\|\gamma(t)-\gamma_{x}(t)\right\|}{\delta\left(\gamma_{x}(t) ; \gamma(t)-\gamma_{x}(t)\right)} \leq \frac{1}{R} .
$$

Since $\gamma$ is a special $x$-curve, we have $k_{D}\left(\gamma(t), \gamma_{x}(t)\right) \rightarrow 0$, and the existence of $t_{R}$ follows from Proposition 1.8.

Moreover, since $p_{x}^{-1}(\gamma(t))$ is the intersection of a complex hyperplane with $D$, we have $\Psi_{t}(\zeta) \in p_{x}^{-1}(\gamma(t))$ and therefore $p_{x}\left(\Psi_{t}(\zeta)\right)=\gamma_{x}(t)=\Psi_{t}(0)$ for all $t>t_{R}$. Hence $t \mapsto \Psi_{t}(\zeta)$ is eventually an $M$-restricted $x$-curve for any $\zeta \in \Delta_{R}$. We can assume without loss of generality that $\tilde{\gamma}_{x}(t) \in K_{\Delta}(1, M)$ for $t>t_{R}$.

Now let $\delta_{0}<1$ be such that $f$ is bounded in any region $T_{z_{0}}\left(x, M, \delta_{0}\right)$. Fix $R>1$; then $\Psi_{t}\left(\Delta_{R / \delta_{0}}\right) \subset D$ for all $t>t_{R / \delta_{0}}$. If $\zeta \in \Delta_{R} \subset \Delta_{R / \delta_{0}}$ we then have

$$
\begin{aligned}
k_{D}\left(\Psi_{t}(\zeta), p_{x}\left(\Psi_{t}(\zeta)\right)\right) & =k_{D}\left(\Psi_{t}(\zeta), \Psi_{t}(0)\right) \leq k_{\Delta_{R / \delta_{0}}}(0, \zeta) \\
& =\omega\left(0, \delta_{0}|\zeta| / R\right)<\omega\left(0, \delta_{0}\right) .
\end{aligned}
$$

Hence $\Psi_{t}\left(\Delta_{R}\right) \subset T_{z_{0}}\left(x, M, \delta_{0}\right)$ for $t>t_{R / \delta_{0}}$. By assumption $|f|$ is bounded, say by $C$, in $T_{z_{0}}\left(x, M, \delta_{0}\right)$. Hence for $R>1$ and $t>t_{R / \delta_{0}}$ we have

$$
\begin{aligned}
k_{\Delta_{c}}\left(f(\gamma(t)), f\left(\gamma_{x}(t)\right)\right) & \leq k_{T_{t_{0}}\left(x, M, \delta_{0}\right)}\left(\gamma(t), \gamma_{x}(t)\right)=k_{T_{\varepsilon_{0}}\left(x, M, \delta_{0}\right)}\left(\Psi_{t}(1), \Psi_{t}(0)\right) \\
& \leq k_{\Delta_{R}}(0,1)=\omega(0,1 / R) .
\end{aligned}
$$

Letting $R \rightarrow+\infty$ we find that as $t \rightarrow 1^{-}$we have

$$
f(\gamma(t)) \rightarrow L \text { if and only if } f\left(\gamma_{x}(t)\right) \rightarrow L .
$$

Now consider $\tilde{f_{x}}=f \circ \varphi_{x} \in \operatorname{Hol}(\Delta, \mathbb{C})$. The map $\tilde{f_{x}}$ is $K$-bounded at 1 because $f$ is $T$-bounded at $x$, and $\zeta=\tilde{p}_{x}\left(\varphi_{x}(\zeta)\right) \in K_{\Delta}(1, M)$ implies $\varphi_{x}(\zeta) \in T_{z_{0}}\left(x, M, \delta_{0}\right)$. Moreover, since $\gamma$ is a $M$-restricted $x$-curve then $\tilde{\gamma}_{x}$ is a non-tangential 1-curve.

By assumption, $f$ has limit $L$ along the special restricted curve $\gamma^{o}$. By (3.1) it follows that $\tilde{f_{x}}$ has limit $L$ along the non-tangential 1-curve $\tilde{\gamma}_{x}^{o}$; then, by the classical Lindelöf principle, we have $\tilde{f}_{x}\left(\tilde{\gamma}_{x}(t)\right)=f\left(\gamma_{x}(t)\right) \rightarrow L$ as $t \rightarrow 1^{-}$for all restricted $x$-curve $\gamma$. But then (3.1) yields $f(\gamma(t)) \rightarrow L$ for all special restricted $x$-curve $\gamma$, and we are done.

\section{The Julia-Wolff-Carathéodory theorem}

In this section we shall deal with the promised version of the Julia-Wolff-Carathéodory theorem in convex domains of finite type. Let $f: D \rightarrow \Delta$ be a bounded 
holomorphic function defined on a bounded domain $D$, and fix $z_{0} \in D$. We shall say that $f$ is $\alpha$-Julia at $x \in \partial D$ if

$$
\liminf _{w \rightarrow x}\left[k_{D}\left(z_{0}, w\right)-\omega(0, f(w))\right]=\frac{1}{2} \log \alpha<+\infty .
$$

This is the basic assumption; from this we shall be able to infer the existence of the (restricted) $K$-limit of $f$ and its (weighted) derivates at $x$.

The existence of the limit for $f$ is a standard consequence of the general Julia lemma for complete hyperbolic domains:

THEOREM 4.1. Let $D \subset \subset \mathbb{C}^{n}$ be a complete hyperbolic domain. Fix $z_{0} \in D$, and let $f \in \operatorname{Hol}(D, \Delta)$ be $\alpha$-Julia at $x \in \partial D$. Then $f$ has $K$-limit $\tau \in \partial \Delta$ at $x$.

ProOF. See [3, Proposition 2.7.15].

If $v=\left(v_{1}, \ldots, v_{n}\right) \in \mathbb{C}^{n}$ is different from the origin, we shall write

$$
\frac{\partial f}{\partial v}(z)=d f_{z}(v)=\sum_{j=1}^{n} v_{j} \frac{\partial f}{\partial z_{j}}(z) .
$$

Our aim is then to prove the following version of the Julia-Wolff-Carathéodory theorem:

THEOREM 4.2. Let $D \subset \subset \mathbb{C}^{n}$ be a convex domain of finite type solid in $z_{0} \in D$, and take $x \in \partial D$ such that there exists the radial limit of $\varphi_{x}^{\prime}$ at 1 . Let $f \in \operatorname{Hol}(D, \Delta)$ be $\alpha$-Julia at $x$. For $v \in \mathbb{C}^{n}, v \neq 0$, let $0<s \leq 1$ be such that $\delta(z)^{s} \kappa_{D}(z ; v)$ is $T$-bounded at $x$. Then the function $\delta(z)^{s-1} \partial f(z) / \partial v$ is $T$-bounded and has restricted $K$-limit at $x$. Furthermore, if $v$ is transversal to $T_{x}^{\mathrm{C}}(\partial D)$ we can take $s=1$, and the limit is zero if and only if $v \in T_{x}^{\mathbf{C}}(\partial D)$.

Such an $s$ always exists. Indeed we have the following

PROPOSITION 4.3. Let $D \subset \subset \mathbb{C}^{n}$ be a convex domain, and let $x \in \partial D$ be such that $\partial D$ is smooth near $x$, and assume that the line type of $x$ is $L \geq 2$. Then there is a neighbourhood $U$ of $x$ such that for any $z \in U \cap D$ and for any $v \in \mathbb{C}^{n}$ different from the origin we have:

(i) $\delta(z) \kappa_{D}(z ; v) \lesssim\|v\|$;

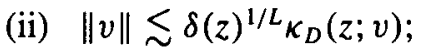

(iii) if $v$ is transversal to $T_{x}^{\mathrm{C}}(\partial D)$ then $\|v\| \lesssim \delta(z) \kappa_{D}(z ; v)$;

(iv) if $v \in T_{x}^{\mathrm{C}}(\partial D)$ then we can find $1 / L \leq s \leq 1-1 / L$ such that $\delta(z)^{s} \kappa_{D}(z ; v)$ is $T$-bounded at $x$. 
PROOF. (i) and (ii) follows from Corollary 1.7, while (iii) follows from Lemma 1.3 (ii). Concerning (iv), if $z \in T_{z_{0}}(x, M, \delta) \cap U$, by Proposition 1.8, we have that

$$
\begin{aligned}
\left\|z-p_{x}(z)\right\| & \lesssim k_{D}\left(p_{x}(z), z\right) \delta\left(p_{x}(z) ; z-p_{x}(z)\right) \lesssim \delta\left(p_{x}(z) ; z-p_{x}(z)\right) \\
& \lesssim \delta\left(p_{x}(z)\right)^{1 / L} \lesssim \delta(z)^{1 / L},
\end{aligned}
$$

because $k_{D}\left(z, p_{x}(z)\right)<\omega(0, \delta)$ implies $\delta\left(p_{x}(z)\right) \lesssim \delta(z)$. Moreover, since $v \in T_{x}^{\mathrm{C}}(\partial D)$,

$$
\begin{aligned}
\left\|v_{N}\right\| & =\left\|\left(v, \mathbf{n}_{z}\right)\right\|=\left\|\left(v, \mathbf{n}_{z}-\mathbf{n}_{x}\right)\right\| \lesssim\|z-x\| \\
& \leq\left\|z-p_{x}(z)\right\|+\left\|p_{x}(z)-x\right\| \lesssim \delta(z)^{1 / L}+\delta(z) \lesssim \delta(z)^{1 / L},
\end{aligned}
$$

where we used the fact that $p_{x}(z)$ approaches $x$ non-tangentially, and thus we have $\left\|p_{x}(z)-x\right\| \lesssim \delta\left(p_{x}(z)\right) \lesssim \delta(z)$. Therefore, by Lemma 1.2,

$$
\kappa_{D}(z ; v) \lesssim \frac{\left\|v_{N}\right\|}{\delta(z)}+\frac{\left\|v_{T}\right\|}{\delta(z)^{1 / 2}} \lesssim \frac{1}{\delta(z)^{1-1 / L}}+\frac{1}{\delta(z)^{1 / 2}} \lesssim \frac{1}{\delta(z)^{1-1 / L}} .
$$

So in the statement of Theorem 4.2 we can take $s=1$ if $v$ is transversal to $T_{x}^{\mathrm{C}}(\partial D)$, and $1 / L \leq s \leq 1-1 / L<1$ otherwise.

The first step in the proof consists in replacing $\delta(z)$ by $1-\tilde{p}_{x}(z)$. This is possible because of the following

LEMMA 4.4. Let $D \subset \subset \mathbb{C}^{n}$ be a convex domain, and fix $z_{0} \in D$. Let $x \in \partial D$ be such that there is a complex geodesic $\varphi_{x}$ with $\varphi_{x}(0)=z_{0}$ and $\varphi_{x}(1)=x$. Then both $\left|1-\tilde{p}_{x}(z)\right| / \delta(z)$ and its inverse are $T$-bounded at $x$ (for any girth $\left.\delta\right)$.

ProOF. Take $z \in T_{z_{0}}(x, M, \delta)$. Then

$$
\left|1-\tilde{p}_{x}(z)\right| \leq M\left(1-\left|\tilde{p}_{x}(z)\right|\right)=M\left(1-K_{z_{0}}\left(p_{x}(z)\right)\right),
$$

where $K_{z_{0}}(z)=\tanh k_{D}\left(z_{0}, z\right)$. On the other hand,

$$
0 \leq k_{D}\left(z_{0}, z\right)-k_{D}\left(z_{0}, p_{x}(z)\right) \leq k_{D}\left(z, p_{x}(z)\right)<\omega(0, \delta),
$$

and thus $1-K_{z_{0}}\left(p_{x}(z)\right) \leq 2 e^{-2 k_{D}\left(z_{0}, z\right)}(1+\delta) /(1-\delta)$. The $T$-boundedness of $\left|1-\tilde{p}_{x}(z)\right| / \delta(z)$ then follows from Lemma 1.3 (i).

Finally, the (global) boundedness of $\delta(z) /\left|1-\tilde{p}_{x}(z)\right|$ follows from Lemma 1.1 (i) and $k_{D}\left(z_{0}, z\right) \geq \omega\left(0, \tilde{p}_{x}(z)\right)$.

Since either $s=1$ or the limit is zero (and every special restricted $x$-curve is eventually inside a $T$-region), we are then left to prove that

$$
\left(1-\tilde{p}_{x}(z)\right)^{s-1} \frac{\partial f}{\partial v}(z)
$$


has the stated restricted $K$-limit; the advantage now is that we deal with a holomorphic function, and so we can apply the Lindelöf principle proved in the last section.

Our first aim then is to prove that (4.1) is $T$-bounded. For this we need the following

LEMMA 4.5. Let $D \subset \subset \mathbb{C}^{n}$ be a convex domain, and fix $z_{0} \in D$. Let $x \in \partial D$ be such that there is a complex geodesic $\varphi_{x}$ with $\varphi_{x}(0)=z_{0}$ and $\varphi_{x}(1)=x$. Then for every $z \in T_{z_{0}}(x, M, \delta), r \in(0,1)$ and complex geodesic $\psi$ such that $\psi(0)=z$ we have $\psi\left(\Delta_{r}\right) \subset T_{z_{0}}\left(x, M_{r}, \delta_{r}\right)$, where $M_{r}=M(1+r) /(1-r)$ and

$$
\delta_{r}=\frac{\delta(1-r)^{2}-2(1-\delta) r}{(1-r)^{2}+2(1-\delta) r} .
$$

PROOF. We have

$$
\begin{aligned}
k_{D}\left(\psi(\zeta), p_{x}(\psi(\zeta))\right) & \leq k_{D}(z, \psi(\zeta))+k_{D}\left(z, p_{x}(z)\right)+k_{D}\left(p_{x}(z), p_{x}(\psi(\zeta))\right) \\
& \leq k_{D}\left(z, p_{x}(z)\right)+2 k_{D}(z, \psi(\zeta)) \\
& <\omega(0, \delta)+2 \omega(0, r)=\omega\left(0, \delta_{r}\right) .
\end{aligned}
$$

Next,

$$
\begin{aligned}
\lim _{t \rightarrow 1}[\omega & \left.\left(\tilde{p}_{x}(\psi(\zeta)), t\right)-\omega(0, t)\right]+\omega\left(0, \tilde{p}_{x}(\psi(\zeta))\right) \\
\leq & \lim _{t \rightarrow 1}\left[\omega\left(\tilde{p}_{x}(z), t\right)-\omega(0, t)\right]+\omega\left(0, \tilde{p}_{x}(z)\right)+2 \omega\left(\tilde{p}_{x}(z), \tilde{p}_{x}(\psi(\zeta))\right) \\
< & \log M+2 k_{D}(z, \psi(\zeta))<\log M_{r}
\end{aligned}
$$

and we are done.

PROPOSITION 4.6. Let $D \subset \subset \mathbb{C}^{n}$ be a convex domain of finite type, solid in $z_{0} \in D$, and take $x \in \partial D$. Let $f \in \operatorname{Hol}(D, \Delta)$ be $\alpha$-Julia at $z_{0}$. Take $v \in \mathbb{C}^{n}$ different from the origin, and choose $0<s \leq 1$ such that $\delta(z)^{s} \kappa_{D}(z ; v)$ is $T$-bounded at $x$. Then (4.1) is $T$-bounded at $x$.

Proof. For every $z \in D$, let $\psi_{z} \in \operatorname{Hol}(\Delta, D)$ be a complex geodesic such that $\psi_{z}(0)=z$ and $\psi_{2}^{\prime}(0)=v / \kappa_{D}(z ; v)$. Let $\delta_{0}>0$ be such that $\delta(z)^{s} \kappa_{D}(z ; v)$ is bounded in $T$-regions of vertex $x$ and girth $\delta_{0}$, fix $0<\delta<\delta_{0}$ and choose $r \in(0,1)$ such that $\delta_{r}<\delta_{0}$, where $\delta_{r}$ is given by (4.2). We claim that (4.1) is bounded in $T$-regions of vertex $x$ and girth $\delta$.

Write

$$
\begin{aligned}
\left(1-\tilde{p}_{x}(z)\right)^{s-1} \frac{\partial f}{\partial v}(z) & =\left(1-\tilde{p}_{x}(z)\right)^{s-1} \kappa_{D}(z ; v)\left(f \circ \psi_{z}\right)^{\prime}(0) \\
& =\left(1-\tilde{p}_{x}(z)\right)^{s-1} \frac{\kappa_{D}(z ; v)}{2 \pi i} \int_{|\zeta|=r} \frac{f\left(\psi_{z}(\zeta)\right)}{\zeta^{2}} d \zeta
\end{aligned}
$$




$$
\begin{aligned}
= & \frac{1}{2 \pi} \int_{0}^{2 \pi} \frac{f\left(\psi_{z}\left(r e^{i \theta}\right)\right)-\tau}{\tilde{p}_{x}\left(\psi_{z}\left(r e^{i \theta}\right)\right)-1} \frac{\tilde{p}_{x}\left(\psi_{z}\left(r e^{i \theta}\right)\right)-1}{\tilde{p}_{x}(z)-1} \\
& \times\left(\frac{\tilde{p}_{x}(z)-1}{\delta(z)}\right)^{s} \frac{\delta(z)^{s} \kappa_{D}(z ; v)}{r e^{i \theta}} d \theta,
\end{aligned}
$$

where $\tau \in \partial \Delta$ is given by Theorem 4.1. We must show that the four factors in the integrand are $T$-bounded.

The fourth factor is $T$-bounded by assumption, and Lemma 4.4 shows that the third factor is $T$-bounded too. Now take $z \in T_{z_{0}}(x, M, \delta)$; then Lemma 4.5 yields

$$
\left|\frac{1-\tilde{p}_{x}\left(\psi_{z}\left(r e^{i \theta}\right)\right)}{1-\tilde{p}_{x}(z)}\right| \leq M_{r} \frac{1-K_{z_{0}}\left(p_{x}\left(\psi_{z}\left(r e^{i \theta}\right)\right)\right)}{1-K_{z_{0}}\left(p_{x}(z)\right)},
$$

where $M_{r}=M(1+r) /(1-r)$. Now,

$$
\begin{aligned}
\frac{1}{2} \log \frac{1-K_{z_{0}}\left(p_{x}\left(\psi_{z}\left(r e^{i \theta}\right)\right)\right)}{2\left(1-K_{z_{0}}\left(p_{x}(z)\right)\right)} & \leq\left|k_{D}\left(z_{0}, p_{x}(z)\right)-k_{D}\left(z_{0}, p_{x}\left(\psi_{z}\left(r e^{i \theta}\right)\right)\right)\right| \\
& \leq k_{D}\left(p_{x}(z), p_{x}\left(\psi_{z}\left(r e^{i \theta}\right)\right)\right) \leq k_{D}\left(z, \psi_{z}\left(r e^{i \theta}\right)\right) \\
& =\omega(0, r),
\end{aligned}
$$

and thus the second factor is $T$-bounded too.

We are left with the first factor. Take $z \in T_{z_{0}}\left(x, M, \delta_{0}\right)$, and set

$$
(1 / 2) \log R=\log M-\omega\left(0, \tilde{p}_{x}(z)\right) \text {. }
$$

In particular, $\tilde{p}_{x}(z) \in E_{\Delta}(1, R)$ and

$$
R \leq M^{2} \frac{1-\left|\tilde{p}_{x}(z)\right|}{1+\left|\tilde{p}_{x}(z)\right|} \leq M^{2}\left|1-\tilde{p}_{x}(z)\right| .
$$

Now, by Lemma 2.11 we know that $f \circ \varphi_{x}$ is $\alpha$-Julia at 1 . Thus $f\left(p_{x}(z)\right) \in E_{\Delta}(\tau, \alpha R)$ and

$$
\begin{aligned}
\log \frac{|\tau-f(z)|}{1+|f(z)|} & =\lim _{t \rightarrow 1}[\omega(f(z), t \tau)-\omega(0, t \tau)]-\omega(0, f(z)) \\
& \leq 2 \lim _{t \rightarrow 1}[\omega(f(z), t \tau)-\omega(0, t \tau)] \\
& \leq 2 \lim _{t \rightarrow 1}\left[\omega\left(f\left(p_{x}(z)\right), t \tau\right)-\omega(0, t \tau)\right]+2 \omega\left(f(z), f\left(p_{x}(z)\right)\right) \\
& \leq \log \left(\alpha R \frac{1+\delta_{0}}{1-\delta_{0}}\right) .
\end{aligned}
$$

Therefore $\left|(\tau-f(z)) /\left(1-\tilde{p}_{x}(z)\right)\right| \leq 2 \alpha M^{2}\left(1+\delta_{0}\right) /\left(1-\delta_{0}\right)$ for all $z \in T_{z_{0}}\left(x, M, \delta_{0}\right)$. Recalling Lemma 4.5 we then get

$$
\left|\frac{\tau-f\left(\psi_{z}\left(r e^{i \theta}\right)\right)}{1-\tilde{p}_{x}\left(\psi_{z}\left(r e^{i \theta}\right)\right)}\right| \leq 2 \alpha M_{r}^{2} \frac{1+\delta_{0}}{1-\delta_{0}}
$$


for all $z \in T_{z_{0}}(x, M, \delta)$, and so the first factor is $T$-bounded too.

We must now prove that (4.1) has limit along a special restricted $x$-curve: the obvious candidate is $\gamma(t)=\varphi_{x}(t)$. We first deal with the complex tangential directions:

PROPOSITION 4.7. Let $D \subset \subset \mathbb{C}^{n}$ be a convex domain of finite type, solid in $z_{0} \in D$, and take $x \in \partial D$. Let $f \in \operatorname{Hol}(D, \Delta)$ be $\alpha$-Julia at $z_{0}$. Take $v \in T_{x}^{\mathrm{C}}(\partial D)$ different from the origin, and choose $0<s<1$ such that $\delta(z)^{s} \kappa_{D}(z ; v)$ is T-bounded at $x$. Then $\lim _{t \rightarrow 1}(1-t)^{s-1} \partial f\left(\varphi_{x}(t)\right) / \partial v=0$.

PROOF. Let $\Phi: \Delta \times \mathbb{C} \rightarrow \mathbb{C}^{n}$ be given by $\Phi(\zeta, \eta)=\varphi_{x}(\zeta)+\eta v$, and set $\Phi^{-1}(D)=B$. Define $\tilde{h}: B \rightarrow \Delta$ by putting $\tilde{h}=f \circ \Phi$. Clearly, $\tilde{h}(\zeta, 0)=f\left(\varphi_{x}(\zeta)\right)$ and $\partial \tilde{h}(\zeta, 0) / \partial \eta=d f_{\varphi_{x}(\zeta)}(v)$. Then we can write

$$
\tilde{h}(\zeta, \eta)=f\left(\varphi_{x}(\zeta)\right)+\eta d f_{\varphi_{x}(\zeta)}(v)+o(|\eta|) .
$$

Set $h(\zeta, \eta)=f\left(\varphi_{x}(\zeta)\right)+(1 / 2) \eta d f_{\varphi_{x}(\zeta)}(v)$; being the average of the first two partial sums of the power series expansion of $\tilde{h}$, we still have $h(B) \subset \Delta$. Writing

$$
h(\zeta, \eta)=f\left(\varphi_{x}(\zeta)\right)+\eta(1-\zeta)^{1-s} g(\zeta)
$$

with $g(\zeta)=d f_{\varphi_{x}(v)}(v) / 2(1-\zeta)^{1-s}$, the assertion is equivalent to $\lim _{t \rightarrow 1} g(t)=0$.

Now, since $t \mapsto \varphi_{x}(t)$ is a non-tangential $x$-curve, and recalling Lemma 1.5 , we see that $B$ contains a domain of the form

$$
E\left(t_{0}\right)=\left\{(\zeta, \eta) \in B \mid \frac{\left|\zeta-t_{0}\right|^{2}}{\left(1-t_{0}\right)^{2}}+\frac{|\eta|^{1 / s_{0}}}{c\left(t_{0}\right)}<1\right\}
$$

for suitable $t_{0}$ close enough to 1 and $c\left(t_{0}\right) \in \mathbb{R}$ (possibly large), where $s_{0}=\min \{1 / 2, s\}$.

Fix $a>1$, and set $\zeta_{t}=t+i a(1-t)$; clearly $\zeta_{t} \in \Delta$ for $t$ close to 1 . Furthermore, we have

$$
1-t<2 \frac{1-t_{0}}{1+a^{2}} \quad \Longrightarrow \quad\left(\zeta_{t}, 0\right) \in E\left(\dot{t_{0}}\right)
$$

and

$$
1-t<\frac{1-t_{0}}{1+a^{2}} \Longrightarrow 1-\frac{\left|\zeta_{t}-t_{0}\right|^{2}}{\left(1-t_{0}\right)^{2}}>\frac{1-t}{1-t_{0}}
$$

Therefore for every $t$ close enough to 1 we can find $\eta_{t} \in \mathbb{C}$ such that

$$
1-\frac{\left|\zeta_{t}-t_{0}\right|^{2}}{\left(1-t_{0}\right)^{2}}>\frac{\left|\eta_{t}\right|^{1 / s_{0}}}{c\left(t_{0}\right)}>\frac{1-t}{1-t_{0}}
$$


In particular, $\left(\zeta_{t}, \eta_{t}\right) \in B$, and we can also assume that $\eta_{t}\left(1-\zeta_{t}\right)^{1-s} g\left(\zeta_{t}\right) \in \mathbb{R}$. Notice moreover that $\left|\eta_{t}\right| \geq c_{1}(1-t)^{s_{0}}$, where $c_{1}$ depends only on $t_{0}$ and not on $a$. We then have $\left|1-\zeta_{t}\right| \geq a(1-t)$ and

$$
\eta_{t}\left(1-\zeta_{t}\right)^{1-s} g\left(\zeta_{t}\right) \geq\left|\eta_{t}\right| a^{1-s}(1-t)^{1-s}\left|g\left(\zeta_{t}\right)\right| \geq c_{1} a^{1-s}(1-t)^{1-s+s_{0}}\left|g\left(\zeta_{t}\right)\right| .
$$

Now, $\zeta_{t}$ eventually belongs to a Stolz region in $\Delta$; hence Lemma 2.11 and the classical one-variable Julia-Wolff-Carathéodory theorem yields

$$
\frac{1-f\left(\varphi_{x}\left(\zeta_{t}\right)\right)}{1-\zeta_{t}}=\alpha+o(1)
$$

that is, $f\left(\varphi_{x}\left(\zeta_{t}\right)\right)=1-(\alpha+o(1))(1-i a)(1-t)$. Thus

$$
\begin{aligned}
1 \geq \operatorname{Re} h\left(\zeta_{t}, \eta_{t}\right) & =1-(\alpha+o(1))(1-t)+\eta_{t}(1-t)^{1-s} g\left(\zeta_{t}\right) \\
& \geq 1-(\alpha+o(1))(1-t)+c_{1} a^{1-s}(1-t)^{1-s+s_{0}}\left|g\left(\zeta_{t}\right)\right|,
\end{aligned}
$$

that is,

$$
\left|g\left(\zeta_{t}\right)\right| \leq \frac{\alpha+o(1)}{c_{1} a^{1-s}}(1-t)^{s-s_{0}}
$$

If $s>1 / 2$, letting $t \rightarrow 1^{-}$we get $\lim _{t \rightarrow 1^{-}}\left|g\left(\zeta_{t}\right)\right|=0$; if $s \leq 1 / 2$ we instead get $\lim \sup _{t \rightarrow 1}\left|g\left(\zeta_{t}\right)\right| \leq \alpha / c_{1} a^{1-s}$. This means that choosing $a$ large enough we can make this lim sup as small as we please; since we can clearly obtain the same estimate for $\zeta_{t}^{\prime}=t-i c(1-t)$, and $g$ is $T$-bounded, it follows that $\lim _{t \rightarrow 1}|g(t)|=0$ in all cases, as claimed.

And finally the transversal directions:

PROPOSITION 4.8. Let $D \subset \subset \mathbb{C}^{n}$ be a convex domain of finite type, solid in $z_{0} \in D$, and take $x \in \partial D$ such that there exists the radial limit of $\varphi_{x}^{\prime}$ at 1 . Let $f \in \operatorname{Hol}(D, \Delta)$ be $\alpha$-Julia at $x$. Then for any $v \in \mathbb{C}^{n}$ transversal to $T_{x}^{\mathrm{C}}(\partial D)$ the limit

$$
\lim _{t \rightarrow 1} \frac{\partial f}{\partial v}\left(\varphi_{x}(t)\right)
$$

exists and it is non-zero.

PROOF. Let $v_{x}=\lim _{t \rightarrow 1} \varphi_{x}^{\prime}(t)$; we know that $v_{x}$ is transversal to $T_{x}^{\mathrm{C}}(\partial D)$. Furthermore,

$$
\frac{\partial f}{\partial \nu_{x}}\left(\varphi_{x}(t)\right)=d f_{\varphi_{x}(t)}\left(v_{x}\right)=\left(f \circ \varphi_{x}\right)^{\prime}(t)+d f_{\varphi_{x}(t)}\left(v_{x}-\varphi_{x}^{\prime}(t)\right)
$$


Now, by Proposition 4.6, $\left\|d f_{\varphi_{x}(t)}\right\|$ (the operator norm) is bounded, and thus the classical one-variable Julia-Wolff-Carathéodory theorem applied to $f \circ \varphi_{x}$ yields

$$
\lim _{t \rightarrow 1} \frac{\partial f}{\partial v_{x}}\left(\varphi_{x}(t)\right)=\alpha .
$$

Since every vector transversal to $T_{x}^{\mathrm{C}}(\partial D)$ is of the form $\lambda v_{x}+w$ for suitable $\lambda \in \mathbb{C}^{*}$ and $w \in T_{x}^{\mathrm{C}}(\partial D)$, from Proposition 4.7 we get the assertion.

REMARK. The hypothesis on the existence of the radial limit of $\varphi_{x}^{\prime}$ is satisfied, for instance, in real-analytic convex domains of finite type (because Lempert's argument [L] applies word by word to prove that complex geodesics extend real-analytic to the boundary) and in convex circular domains (because $\varphi_{x}(\zeta)=\zeta x$ ). Anyway, Proposition 4.8 holds under weaker hypotheses too. For instance, it suffices to assume that $\left\|\varphi_{x}^{\prime}(t)\right\|$ is bounded as $t$ goes to 1 and write

$$
d f_{\varphi_{x}(t)}\left(\lambda \mathbf{n}_{x}\right)=\left(f \circ \varphi_{x}\right)^{\prime}(t)+d f_{\varphi_{x}(t)}\left(\lambda \mathbf{n}_{x}-\varphi_{x}^{\prime}(t)_{N}\right)-d f_{\varphi_{x}(t)}\left(\varphi_{x}^{\prime}(t)_{T}\right),
$$

where $\lambda=v(1)=\left(\varphi_{x}^{\prime}(1), \mathbf{n}_{x}\right) \neq 0$. The first addendum on the right goes to $\alpha$; the second goes to zero, because $\left\|d f_{\varphi_{x}(t)}\right\|$ is bounded and $\left\|\lambda \mathbf{n}_{x}-\varphi_{x}^{\prime}(t)_{N}\right\| \rightarrow 0$; and the third goes to zero too, because $\left\|d f_{\varphi_{x}(r)}\right\|=o(1)$ when restricted to complex tangential vectors. Thus we have again the existence of the limit (4.3) for one transversal vector, and therefore for all of them.

So we finally have both $T$-boundedness and the existence of the limit along a special restricted $x$-curve for (4.1); applying the Lindelöf principle Theorem 3.2, and recalling Lemma 4.4, we get Theorem 4.2.

\section{References}

[1] M. Abate, 'Boundary behaviour of invariant distances and complex geodesics', Atti Accad. Naz. Lincei Rend. Cl. Sci. Fis. Mat. Natur. (8) 80 (1986), 100-106 (1987).

[2] — 'Common fixed points of commuting holomorphic maps', Math. Ann. 283 (1989), 645-655.

[3] - Iteration theory of holomorphic maps on taut manifolds (Mediterranean Press, Rende, 1989).

[4] - 'The Lindelöf principle and the angular derivative in strongly convex domains', J. Analyse Math. 54 (1990), 189-228.

[5] — 'Angular derivatives in strongly pseudoconvex domains', in: Several complex variables and complex geometry, Part 2 (Santa Cruz, CA, 1989) (Amer. Math. Soc., Providence, RI, 1991) pp. $23-40$.

[6] — 'The Julia-Wolff-Carathéodory theorem in polydisks', J. Anal. Math. 74 (1998), 275-306.

[7] M. Abate and R. Tauraso, 'The Julia-Wolff-Carathéodory theorem(s)', in: Complex geometric analysis in Pohang (1997) (Amer. Math. Soc., Providence, RI, 1999) pp. 161-172. 
[8] C. Carathéodory, 'Über die winkelderivierten von beschränkten analytischen Funktionen', Sitzungsber. Preuss. Akad. Wiss., Berlin (1929), 39-54.

[9] C.-H. Chang, M.-C. Hu and H.-P. Lee, 'Extremal analytic discs with prescribed boundary data', Trans. Amer. Math. Soc. 310 (1988), 355-369.

[10] J. A. Cima and S. G. Krantz, 'The Lindelöf principle and normal functions of several complex variables', Duke Math. J. 50 (1983), 303-328.

[11] E. M. Čirka, 'The Lindelöf and Fatou theorems in C', Math. USSR-Sb. 21 (1973), 619-641.

[12] P. Fatou, 'Séries trigonométriques et séries de Taylor', Acta Math. 30 (1906), 335-400.

[13] F. Forstneric and J.-P. Rosay, 'Localization of the Kobayashi metric and the boundary continuity of proper holomorphic mappings', Math. Ann. 279 (1987), 239-252.

[14] G. H. Hardy and J. E. Littlewood, 'Some properties of fractionals integrals, Il', Math. Z. 34 (1932), 403-439.

[15] M. Hervé, 'Quelques propriétés des applications analytiques d'une boule à $m$ dimensions dans elle-même', J. Math. Pures Appl. 42 (1963), 117-147.

[16] F. Jafari, 'Angular derivatives in polydisks', Indian J. Math. 35 (1993), 197-212.

[17] M. Jarnicki and P. Pfiug, Invariant distances and metrics in complex analysis (Walter de Gruyter, Berlin, 1993).

[18] G. Julia, 'Extension nouvelle d'un lemme de Schwarz', Acta Math. 42 (1920), 349-355.

[19] S. Kobayashi, Hyperbolic complex spaces (Springer, Berlin, 1998).

[20] L. Lempert, 'La métrique de Kobayashi et la représentation des domaines sur la boule', Bull. Soc. Math. France 109 (1981), 427-474.

[21] E. Lindelöf, 'Sur un principe générale de l'analyse et ses applications à la theorie de la représentation conforme', Acta Soc. Sci. Fennicae 46 (1915), 1-35.

[22] J. D. McNeal, 'Convex domains of finite type', J. Funct. Anal. 108 (1992), 361-373.

[23] _ - 'Estimates on the Bergman kernels of convex domains', Adv. Math. 109 (1994), 108-139.

[24] P. R. Mercer, 'Complex geodesics and iterates of holomorphic maps on convex domains in $\mathbf{C}^{\mathbf{n}}$, Trans. Amer. Math. Soc. 338 (1993), 201-211.

[25] H. L. Royden and P. M. Wong, 'Carathéodory and Kobayashi metrics on convex domains', Preprint, 1983.

[26] W. Rudin, Function theory in the unit ball of $\mathbb{C}^{n}$ (Springer, Berlin, 1980).

[27] N. Vormoor, 'Topologische Fortsetzung biholomorphen Funktionen auf dem Rande bei beschränkten streng-pseudokonvexen Gebieten in $C^{n}$ mit $C^{\infty}$ Rand', Math. Ann. 204 (1973), 239-261.

[28] J. Wolff, 'Sur une généralisation d'un théorème de Schwarz', C. R. Acad. Sci. Paris 183 (1926), $500-502$.

Dipartimento di Matematica

Università di Roma 'Tor Vergata'

Via della Ricerca Scientifica

00133 Roma

Italy

e-mail: abate@mat.uniroma2.it, tauraso@mat.uniroma2.it 